\title{
Aging Regulates Post-Viral Asthmatic Airway Pathology
}

\section{Guy Hazan}

Washington University School of Medicine

Anna Eubanks

Washington University School of Medicine

Carrie Gierasch

Washington University in St. Louis

Jeffrey Atkinson

Washington University School of Medicine

Carolyn Fox

Washington University School of Medicine

Ariel Hernandez-Leyva

Washington University School of Medicine

Anne Rosen

Washington University School of Medicine

Andrew Kau

Washington University School of Medicine

https://orcid.org/0000-0002-9386-3766

\section{Eugene Agapov}

Washington University School of Medicine

Jennifer Alexander-Brett

Washington University School of Medicine

Deborah Steinberg

Washington University School of Medicine

\section{Diane Kelley}

Washington University School of Medicine

Michael White

Washington University School of Medicine

Derek Byers

Washington University School of Medicine

\section{Kangyun Wu}

Washington University School of Medicine

Shamus Keeler

Washington University School of Medicine

Yong Zhang 
Washington University School of Medicine

\section{Jeffrey Koenitzer}

Washington University School of Medicine

\section{Michael Holtzman}

Washington University School of Medicine

Jeffrey Haspel ( $\sim$ jhaspel@dom.wustl.edu )

Washington University School of Medicine

\section{Article}

Keywords: asthma, diseases of childhood, immunology

Posted Date: June 1st, 2021

DOl: https://doi.org/10.21203/rs.3.rs-544240/v1

License: (c) (i) This work is licensed under a Creative Commons Attribution 4.0 International License. Read Full License 


\section{Abstract}

Asthma is a common chronic disease of childhood, but for unknown reasons disease activity sometimes subsides as children mature. To understand why, we exposed mice across a range of ages to viral and allergic triggers of asthma exacerbations and airway pathology. We found that pathology induced by Sendai virus (SeV) or influenza A virus (IAV) occurred selectively in juvenile mice in a microbiomeindependent manner, while the same phenotypes induced by allergens were insensitive to age. Age-specific responses to $\mathrm{SeV}$ included a juvenile bias towards type-2 airway inflammation that emerged early in infection and was lost with maturation. With aging, we observed progressive transcriptional changes to alveolar macrophages (AMs) including the acquisition of high-level MHC-II expression. Importantly, depleting AMs canceled the protective effects of maturity on post-viral airway pathology. Thus, aging of the lung-immune microenvironment influences chronic outcomes of respiratory viral infection and may help to explain childhood asthma remission.

\section{Introduction}

Asthma is a common respiratory disease characterized by paroxysms of airway obstruction, airway hyperresponsiveness, and inflammation. Disease activity is triggered by periodic exposures to infectious agents, allergens, or non-specific irritants that induce a stereotypical pattern of airway remodeling ${ }^{1}$. Histologic asthma phenotypes include airway mucous cell metaplasia, peri-bronchial infiltration of immune cells, and airway wall thickening ${ }^{2}$. Although asthma can arise at any age, most patients first present in early childhood (under 6 years-old) ${ }^{3,4}$. Childhood-onset asthma is frequently marked by "type 2 " inflammatory signatures that include airway eosinophilia and the secretion of cytokines like interleukin (IL)-4, IL-5, and IL$13^{5}$.

While asthma represents the most common chronic disease of childhood, lifelong symptoms are not inevitable ${ }^{6}$. Roughly $50 \%$ of children who present with asthma before the age of six will experience remission of their symptoms by early adulthood, and $20-25 \%$ of cases will achieve resolution of their airway hyper-responsiveness by objective testing 7,8 . Across all asthma patients, disease exacerbation rates are highest in children under four years of age and improve gradually with aging, reaching a nadir by early adulthood $^{9}$. Asthma remission is a durable feature of pediatric asthma in populations worldwide, and its incidence is stable from decade to decade, suggesting it is a universal feature of the disease ${ }^{10}$. The biological basis of asthma remission is poorly understood. Research into this phenomenon is hampered by the need for long-term longitudinal cohorts maintained over decades to differentiate remitting childhoodonset asthma cases from those that persist. Critically, we lack animal models that recapitulate aspects of asthma remission and that would enable mechanistic studies. A better understanding of how and why asthma improves with age in some children could yield novel means of controlling or even resolving this disease in all sufferers.

One clue about the origins of asthma remission is that as children mature the seasonality of asthma exacerbations becomes progressively less pronounced ${ }^{11,12}$. This observation might imply a developmental 
process that mutes host responses to seasonal triggers of asthma exacerbations like viruses and allergens and leads to a reduction in airway pathology elicited by these agents. Over time, clinical asthma remission might thus be achieved in developing children.

To explore this idea, we challenged mice across a span of ages with viral and allergic agents associated with asthma exacerbations and which also induce asthmatic airway traits like chronic mucous cell metaplasia, airway inflammation, and airway hyper-responsiveness. Our results suggest that viral but not allergic triggers of asthmatic phenotypes are highly sensitive to the age of the organism, and their ability to elicit chronic airway pathology declines with maturation. Our data also identify a role for alveolar macrophages in mediating the age-related declines in post-viral airway pathology. Our findings have implications for the biological basis of asthma remission in children and identify an animal model for further investigation of this understudied phenomenon.

\section{Results}

Virus-triggered chronic airway disease is age-sensitive. To examine whether age is an important variable in the induction of asthmatic phenotypes, we began by infecting mice of various ages with Sendai virus (SeV; Fig. 1a). SeV is a parainfluenza virus native to rodents that produces an acute bronchiolitis of roughly 2 weeks duration ${ }^{13}$. Chronic airway pathology begins to ensue by 3 weeks post-infection and includes characteristic features of asthma including mucous cell metaplasia, chronic inflammation, and airway hyper-responsiveness ${ }^{13}$. Juvenile ( 6 weeks old) mice infected with SeV developed histological evidence of chronic airway inflammation and mucous cell metaplasia by 49 days post-infection (DPI) as expected, but these phenotypes were greatly attenuated in older (11 months old) mice (Fig. 1b, c). Induction of inflammatory mucus as measured by MUC5AC gene expression was significantly greater in juvenile mice treated with $\mathrm{SeV}$ compared to mature mice across a range of $\mathrm{SeV}$ doses and was independent of sex (Figs. 1d and Supplementary Fig. 1). Similarly, induction of $I L 13$, an important driver of mucus production in the SeV model and in human asthma ${ }^{14}$, occurred selectively in juvenile animals (Fig. 1e). Strong induction of MUC5AC by SeV occurred as long as mice were 3 months old or younger at the time of infection, an age range analogous to the span between childhood and early adulthood in humans ${ }^{15}$ (Fig. 1f). Juvenile mice also developed airway hyper-responsiveness to methacholine by SeV 49 DPI as previously described ${ }^{13}$, but this response failed to materialize in SeV-infected mature mice (Fig. 1g). We next asked whether the age sensitivity of post-viral lung disease observed with $\mathrm{SeV}$ was generalizable to other common respiratory viruses. We infected mice with a strain of H1N1 influenza A virus (IAV) previously shown to provoke chronic lung pathology (Fig. 2a) ${ }^{16}$. While IAV infection produced similar levels of acute viral RNA expression in juvenile and mature mice, production of chronic lung pathology occurred selectively in juvenile mice as assessed by histologic severity, airway hyper-responsiveness, and mucous cell

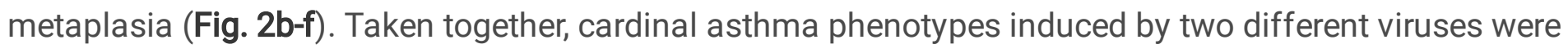
dependent on juvenile age status in mice.

Asthmatic airway phenotypes can also be induced in mice by allergic sensitization ${ }^{17}$. We therefore examined how mouse age affects the production of airway pathology by two model allergens: ovalbumin 
(Ova) and Alternaria alternata extract (Figs. $1 \mathrm{~h}$ and Supplementary Fig. 2$)^{17}$. MUC5AC induction by Ova sensitization was similar in juvenile and mature mice, like prior reports (Fig. $1 \mathrm{~h})^{18}$. In response to Alternaria extract, both juvenile and mature mice developed extensive mucous cell metaplasia and peri-bronchial airway inflammation (Supplementary Fig. 2a, b). While the extent of airway hyper-responsiveness and lung consolidation induced by Alternaria in juvenile mice was greater than in mature mice (Supplementary Fig. 2c, e), both age groups were abnormal compared to PBS-treated controls. Induction of mucous cell metaplasia and MUC5AC expression was similar across age groups (Supplementary Fig. 2d, f).

Additionally, $I L-33$ and $I L-13$, cytokines that drive mucus production in this mode ${ }^{19}$, were similar in juvenile and mature mice (Supplementary Fig. 2f). We concluded that allergen induction of asthmatic phenotypes was relatively age-insensitive in mice, in contrast to viruses.

The physical characteristics of mature $\mathrm{C} 57 \mathrm{BL} / 6 \mathrm{~J}$ mice used in our studies differed from their juvenile counterparts in several respects including increased adiposity and divergent microbiome composition 20,21 . Since both these factors have been shown to regulate inflammatory responses ${ }^{22,23}$, we examined their role in SeV-induced chronic lung pathology. We found that feeding juvenile mice a high fat diet for 6 weeks, enough to induce significant weight gain, did not significantly affect MUC5AC induction at SeV 49 DPI (Supplementary Fig. 3). To evaluate the role of microbiome composition, we added a broad-spectrum antibiotic cocktail (VNAM) to the drinking water of juvenile and mature mice (Fig. 3a). VNAM treatment strongly suppressed stool DNA content and microbial diversity at the time of SeV infection (0 DPI, Fig. 3b, c). While juvenile and mature mice could be differentiated from one another by stool 16S DNA sequencing at baseline, VNAM treatment eliminated clear-cut age differences during and after SeV infection (Fig. 3d). SeV RNA expression was not affected by VNAM during the acute viral illness in either age group (Fig. 3e). At SeV 49 DPI, VNAM treatment also did not alter age-related differences in airway hyper-responsiveness, MUC5AC, IL33, and TREM2 induction (Fig. 3f, g).

Taken together, we found that the ability of age to regulate the production of key asthmatic phenotypes depends on the method of induction. Viral triggers of asthmatic traits were strongly age-dependent, and pathology occurred selectively in the juvenile to young adult age bracket, while allergic triggers of the same phenotypes were largely age-independent. The age sensitivity of virus-induced lung pathology could not be explained by differences in adiposity or microbiome composition between juvenile and mature mice.

Age alters the transcriptional landscape of post-viral airway inflammation. To examine how age affects molecular signatures associated with asthmatic phenotypes, we challenged mice of different ages with $\mathrm{SeV}$ or Ova and then analyzed the resultant chronic lung pathology by bulk RNAseq (Fig. 4a and Supplementary Data 1). We compared gene expression in all treatment groups relative to naïve juvenile mice (Fig. 4b,c), and separately compared remodeled juvenile and mature lungs within each model (Fig. 4d, e). With either analytical approach, Ova-treated lungs exhibited more age-specific differential gene expression than SeV-treated lungs, and there was also limited overlap in age-specific differential gene expression between the two models (Fig. $\mathbf{4 b}$ and d). Focusing on SeV-remodeled lungs, juvenile mice overexpressed markers of alternately activated (M2-type) macrophages relative to mature mice, including CHI3L3/4, RETNLA, and ARG1 (Fig. 4c, e and f) ${ }^{24,25}$. Post-viral juvenile mice also overexpressed epithelial 
cell alarmins relative to mature mice, including IL-33 whose expression localizes to type-II pneumocytes and a subset of airway progenitor cells (Fig. $\mathbf{4 g})^{14,26,27}$. Importantly, IL-33 is critical for IL-13 and MUC5AC induction after SeV infection ${ }^{14}$. Another alarmin overexpressed in post-viral juvenile lungs is KRT17 (Fig. 4e), whose function in asthma was not previously described. However, in the dermatologic literature KRT17 has been implicated in keratinocyte proliferation and migration during skin wound healing, and type 2 inflammatory polarization in the setting of basal cell carcinoma 28,29 . KRT17induction by SeV was temporally distinct from IL33 and was induced 40 to 70-fold at 12 DPI before dropping to a roughly 10-fold induction by 49 DPI (Fig. 4h). Interestingly, KRT17 protein expression in the basal lung was specific to the proximal ends of airway cilia (Fig. 4i, left). By SeV 12-DPI ciliated airway cells were lost and KRT17 expression was pronounced in basal cells engaged in airway epithelial repair (Fig. 4i, middle), which is described to occur at this time point ${ }^{30}$. At SeV 49 DPI, KRT17 expression in ciliated epithelial cells was restored but continued to be expressed by a subset of basal cells within the airway as well as cells outside the airway (Fig. 4i, right). Altogether, genome-wide analysis of post-viral lung pathology revealed a juvenile age-specific signature favoring myeloid and epithelial markers of type 2 inflammation.

Age-specific responses to SeV infection arise early after infection. To identify points of divergence between juvenile and adult mice along the 7-week course of the SeV model (Fig. 1a), we first examined indices of acute bronchiolitis severity (Fig. 5). Weight loss during the acute phase of SeV infection (indicative of systemic illness) followed similar kinetics in juvenile and mature mice, with slightly increased percent weight loss in older mice (Fig. 5a). SeV viral load was also similar across age groups as reflected by viral RNA expression, with only small differences detected during the resolution of acute infection at SeV 8-12 DPI (Fig. 5b). Analysis of bronchioalveolar lavage (BAL) fluid indicated that SeV-infected juvenile mice and mature mice developed similar patterns of airway inflammation as measured by BAL cell count and cell differential, although there was a trend towards higher cellularity in juvenile BALs at SeV 8 DPI (Fig. 5c-f). In contrast to the above similarities, airway mechanics measurements at SeV 5 DPI (the peak of acute illness) showed that juvenile mice exhibited greater methacholine sensitivity than mature mice despite similar levels of viral RNA expression (Fig. 5g). This ventilatory difference diminished at subsequent time points, and by SeV 12 DPI, airway hyper-responsiveness had normalized in both age groups (Fig. $5 \mathrm{~h}$, i). In the setting of acute bronchiolitis, increased methacholine sensitivity might reflect the distribution of small airways disease. We therefore counted SeV-infected airway cells in acutely infected mice and found that the percentage of $\mathrm{SeV}^{+}$cells per airway was greater in juvenile mice than mature mice at 5-8 DPI (Fig. 5j, k). To summarize, while general indices of respiratory illness and viral load were similar across age groups, SeV-infected juvenile mice tended to have a wider distribution of virus-infected airway cells which was associated with transient airway mechanical differences between the age groups. A key insight was that age-specific differences emerged early during SeV infection.

Type 2 cytokine bias in SeV-infected juvenile mice. To investigate age-specific inflammatory patterns in response to $\mathrm{SeV}$, we profiled $\mathrm{BAL}$ cytokines in juvenile and mature mice at various time points after infection (Fig. 6). Viewed as a heat map, the overall time course of BAL cytokine expression was grossly similar in both age groups, with peak expression levels at SeV 5-8 DPI (Fig. 6a). Consistent with this similar time course, principal component analysis did not segregate juvenile from mature cytokine profiles 
at any point post-SeV infection (Fig. 6b). However, when comparing differential expression of individual cytokines (rather than considering the entire panel as an ensemble) juvenile mice expressed a significantly higher amounts of the type 2 cytokines IL-4, -5, and - 13 (Fig. 6c, d). Interestingly, IL-4 and IL-5 expression was biphasic with an initial peak during the acute infection (SeV 5 DPI) and another at 49 DPI once chronic airway disease became established (Fig. 6e, f). Expression of both these cytokines at SeV 49 DPI directly correlated with airway resistance in the chronically remodeled lungs, suggesting these cytokines are important for establishing the airway hyper-responsiveness phenotype in this model (Fig. 6g). These data demonstrate a type 2 cytokine bias in response to $\mathrm{SeV}$ infection that is present in juvenile but not mature mice. As with airway mechanics (Fig. $\mathbf{5 g}$ ), age-specific cytokine responses emerge early if transiently during acute $\mathrm{SeV}$ respiratory illness.

Lung leukocyte composition changes with age. To further clarify immunological differences between juvenile and mature mice throughout $\mathrm{SeV}$ infection, we used mass cytometry to examine whole lung leukocyte composition (Figs. 7 and Supplementary Fig. 4). Principal component analysis revealed that juvenile and mature lungs were most divergent in terms of their leukocyte composition prior to SeV infection (0 DPI) and at 49 DPI when chronic airway disease is established (Fig. 7a). At 0 DPI, mature mouse lungs exhibited greater frequencies of PD $-1^{+}$and $\mathrm{CD} 44^{+} \mathrm{T}$-cells compared to juvenile lungs (Supplementary Fig. 4a, b). Leukocyte infiltration kinetics into SeV-infected lungs could be grouped into 3 general patterns: a "U" or "S"-shaped pattern of infiltration (cluster I), a peak at SeV 5 DPI (cluster II), or a peak at 12 DPI (cluster III) (Fig. 7b). While these trafficking patterns were similar across age groups, the overall prevalence of specific cell types differed with age (Figs. 7c and Supplementary Fig. 4c-j). B-cells and neutrophils were more prevalent in mature mice over discrete intervals during SeV infection, while eosinophils accumulated progressively only in juvenile mice (Fig. 7d-f). Alveolar macrophages (AMs) were unique among the cell types we measured for being more prevalent in juvenile mice at baseline and at SeV $49 \mathrm{DPI}$ (Fig. $\mathbf{7 g}$ ). We therefore scrutinized AMs to further understand how the process of aging affects this cell type.

Alveolar macrophages (AMs) exhibit age-related changes to immunophenotype and gene expression. AMs are the most abundant innate immune cell in normal lungs ${ }^{31}$. As tissue resident macrophages, they play a role in maintaining normal lung airspace homeostasis and, reciprocally, are transcriptionally programmed by the alveolar microenvironment ${ }^{32}$. Importantly, AMs are transiently depleted by acute respiratory viral infections ${ }^{33,34}$. Using visualization of t-distributed stochastic neighbor embedding (viSNE) analysis of mass cytometry data ${ }^{35}$, we noticed that $\mathrm{AMs}$ from juvenile and mature mice congregated in distinct clusters that were strongly influenced by differential surface expression of MHC-II protein, both at baseline and at SeV 49 DPI (Figs. 8a.b). In mice, AMs are usually defined by low expression of MHC- $-1{ }^{36}$, but we observed significant upregulation of this complex with aging that approached levels seen in dendritic cells (DCs, Fig. 8b). In contrast, MHC-Il expression in DCs was similar across age groups (Fig. 8b). AMs developed $\mathrm{MHC}-\mathrm{II}^{\mathrm{Hi}}$ status progressively with age achieving $>60 \%$ prevalence by 7 months-old (Fig. $8 \mathrm{c}$ ), roughly the age where MUC5AC induction by SeV became noticeably diminished (Fig. 1f). Analysis of permeabilized cells by mass cytometry confirmed that elevated cell surface MHC-II in mature AMs was a function of heightened protein expression (Fig. 8d). Interstitial macrophages also demonstrated increased 
MHC-Il albeit to a lesser extent, while DCs and CD11 $\mathrm{b}^{+}$lung monocytes showed no difference based on age (Fig. 8d). Interestingly, juvenile mice acquired MHC-II ${ }^{\mathrm{Hi}} \mathrm{AMs}$ after SeV infection, although the frequency of these cells in juvenile mice never achieved the levels seen in mature mice infected in parallel (Fig. 8e). To examine whether MHC-II expression correlated with antigen-processing activity, we intranasally instilled DQconjugated ovalbumin particles (DQ-Ova) into juvenile and mature naïve mice (Fig. 8f). DQ-Ova particles are fluorescently quenched but once proteolyzed they produce a green fluorescence ${ }^{37}$. Overall, mature AMs processed DQ-Ova less efficiently than juvenile AMs, and the level of MHC-II surface expression did not correlate with DQ-Ova processing activity (Fig. 8f). These results suggest that MHC-II expression represents a marker of AM aging (or reflects changes to the lung airspace microenvironment with aging) rather than a re-tasking of AMs for antigen presentation in the quiescent state. To examine the transcriptional landscape of AM aging, we performed RNAseq analysis of BAL samples from naïve mice, which are largely composed of AMs (Supplementary Data 2). We observed age-dependent expression of $H 2-A A$ and $H 2-E B 1$, genes that encode the 1A/1E MHC-Il proteins detected by flow cytometry (Fig. 8g). Also upregulated was CIITA, a master regulator of MHC-II gene expression ${ }^{38}$ (Fig. 8g). To put these findings in context, 1024 genes $(2.5 \%$ of identified annotations) demonstrated age-dependent expression in BAL samples from naïve mice using a cutoff of $p<0.05$, with a slight majority $(55 \%)$ demonstrating increased expression in the juvenile $(<3$ months old) compared to the mature ( $>7$ months old) age range (Fig. $8 \mathrm{~h}$ ). However, from an informatic perspective all KEGG pathways found to be enriched in the total set of 1024 genes were entirely driven by the subset whose expression is higher with maturity (Fig. 8i, blue shaded terms). These KEGG terms included "asthma" and multiple pathways connected to antigen presentation, all of which include MHC-II genes in their functional annotation definitions (Fig. 8i). Thus, a major informatic feature of age-associated gene expression in BAL cells is the acquisition of MHC-Il gene expression with aging.

To determine whether MHC-Il upregulation was a specific feature of AM aging, we performed single cell RNAseq on pooled BAL samples from naïve juvenile ( 6 weeks old) and mature ( 12 months old) mice (Fig. 9). UMAP analysis revealed that juvenile and mature mice harbor similar populations of cell types that tightly co-cluster (Fig. 9a). There were subtle differences in the cell differential, with mature BALs containing greater amounts of lymphocytes (Fig. 9b). We noted a higher cellular yield and a higher prevalence of mitotically active $\left(K I-67^{+}\right)$AMs in BALs from juvenile mice compared to mature mice, consistent with prior reports (Fig. 9b) ${ }^{39}$. The age-dependent upregulation of $H 2-A A$ seen by bulk RNAseq was confirmed by single cell analysis and mapped broadly across the entire AM cluster in mature mice (Fig. 9c). Examining global AM gene expression by scRNAseq revealed a distinct group of genes upregulated in mature compared to juvenile AMs that were predominantly MHC-II pathway genes (Fig. 9d, blue filled symbols). Age-regulated expression of these genes was specific to AMs and was not observed in the other cell types detected in BALs (Fig. 9E, arrow). Thus, scRNAseq confirmed differential MHC-II gene expression to be a prominent and selective informatic feature of $A M$ aging in healthy mice. We should note that a recent report compared single cell gene expression in 4-6 months-old versus 18-24 months-old AMs sorted by flow cytometry but did not observe differences in MHC-II gene expression aside from CD74 (also found in our dataset $)^{40}$. However, this analysis left out juvenile mice where the major differences in MHC-II gene expression occur, and employed cells exposed to the stress of tissue dissociation and cell sorting. 
We considered the possibility that AMs from mature animals might originate from bone marrow-derived monocytes as opposed to the embryonic yolk sac, and this alternate origin might explain differences $\mathrm{MHC}-\mathrm{II}$ expression. However, AMs from healthy mature mice exclusively expressed markers connoting embryonic but not bone marrow origin (Figs. 9f ${ }^{41}$. Altogether, our data demonstrate substantial AM programming with aging in terms of the number of these cells in the lung, global gene expression, antigen processing potential, and MHC-II class gene expression. Importantly, gene expression changes to AMs plateau by 7 months of age, indicating the phenomenon is one of organism maturation rather than senescence or old age.

AM depletion modifies post-viral lung pathology. To examine whether AMs mediate the age-dependency of post-viral asthma phenotypes, we employed clodronate liposomes to selectively deplete AMs prior to SeV challenge ${ }^{41}$ (Fig. 10a). Intranasal clodronate liposomes substantially reduced AM abundance and normalized differences in AM number between juvenile and mature mice (Fig. 10b, c). However, neither clodronate nor control liposomes were immunologically silent interventions. Clodronate treatment stimulated airspace neutrophil influx, presumably to clear cellular debris from dead AMs (Fig. 10b). Additionally, both treatments elevated BAL IL-6 levels regardless of mouse age (Supplementary Fig. 5a). As such, clodronate treatment modified the lung immune microenvironment in addition to simply depleting AMs. Nonetheless, neither clodronate nor control liposome treatment produced chronic lung pathology by themselves (Supplementary Fig. 5b). Clodronate liposomes enhanced the severity of SeV infection compared to control liposomes as judged by survival regardless of age, but maximum weight loss among survivors was similar (Supplementary Fig. 5c, d). SeV RNA expression at 5 DPI was also unaffected by clodronate or control liposomes and remained insensitive to mouse age (Supplementary Fig. 5e). In controlliposome treated mice, expression of asthma phenotypes at SeV 49 DPI occurred selectively in juvenile mice, including airway pathology, mucous cell metaplasia, MUC5AC and IL 13 expression, as well as airway hyper-responsiveness (Fig. 10d-h). In contrast, clodronate-treated mice displayed abnormal airway pathology in both juvenile and mature age groups at SeV $49 \mathrm{DPI}$ as measured by histology and airway hyper-responsiveness (Fig. 10d-f). Clodronate treatment also suppressed age differences in MUC5AC and IL-13 expression at SeV 49 DPI (Fig. 10g, h). Collectively our data suggest that a specific lung immune microenvironment and AM constituency is required to protect mice from post-viral lung pathology, and that this microenvironment is acquired through normal developmental aging.

\section{Discussion}

For many chronic inflammatory diseases, the optimal goal of medical therapy is remission $42,43,44,45$. Yet, current asthma management guidelines focus almost exclusively on control of symptoms ${ }^{46,47}$, partly because the biological mechanisms underlying asthma remission are obscure. Here, our data suggest a novel theory for asthma remission based on the differential effects of age on viral and allergic triggers of the disease. Specifically, we identified in mice an age-dependent response to respiratory viruses that regulates whether an acute viral infection will lead to chronic type 2 airway inflammation. In contrast, allergic triggers of the same airway pathology were much less sensitive to the chronological age. The decline in post-viral pathology mapped to the juvenile-to-adult age transition, which mirrors the 
phenomenon of asthma remission in children. On a molecular level, mature age was marked by a lower propensity towards type 2 inflammatory responses after viral challenge, a change in the distribution of infection within the bronchial tree, and an altered immune micro-environment in the lung at baseline as reflected by AM number and gene expression. Extrapolated to childhood-onset asthma, our data frames asthma remission as an evolution in the host response to viral triggers, leading to progressively fewer asthma exacerbations as some children approach adulthood.

Our observations in mice correlate well with what is known about the epidemiology of asthma remission in children $8,48,49$. When asthma arises in childhood, it usually presents as an acute wheezing illness, often triggered by common respiratory viruses like RSV, rhinovirus C, influenza, and parainfluenza ${ }^{50}$. Rates of asthma remission in children tend to plateau roughly 10-15 years after disease onset, but the likelihood that remission will ever occur correlates inversely with age of asthma debut ${ }^{51}$. This relationship suggests that the pathophysiology usually associated with early-life asthma symptoms- viral infection- may also be connected to the process of remission. In support of this, seasonal peaks in asthma exacerbations become progressively less prominent as children age ${ }^{11}$. Of note, asthma seasonality correlates with the seasonality of respiratory viral infection in children, but this relationship breaks down by early adultood 52 , 53,54 . Thus, epidemiological data implies that responses to viral triggers of asthma must evolve during childhood, as predicted by our observations in mice. In contrast to viruses, atopy is strong negative predictor of asthma remission and the resolution of asthmatic airway remodeling $8,48,51,55,56$. As such, the dichotomy we observed between viral versus allergic inducers of asthma phenotypes in mice mirrors asthma remission predictors in patients. Why developmental aging should impact virus-host responses differently than allergen-host responses is unclear but deserves further investigation. It is tempting to speculate that the dichotomy reflects an evolutionary need to limit lung injury in the face of yearly cycles of respiratory viral infection that, due to antigenic shifts, cannot entirely be forestalled by acquired immunity. Recently, molecular biomarkers of pediatric asthma remission have garnered increased attention with one genome-wide and one epigenome-wide association study published so far ${ }^{57,58}$. Importantly, these studies identified a polymorphism in the IL-33 receptor IL 1R1 that was associated with asthma remission by adulthood $^{58}$. IL-33 is a major contributor to the type 2 inflammatory response in asthma and emerged in our data as an age-sensitive predictor of asthma phenotypes after $\mathrm{SeV}$ infection. This concordance further underscores the value of the $\mathrm{SeV}$ model in understanding mechanisms driving asthma remission, which owing to the nature of the phenomenon are extremely difficult to investigate in human subjects.

Our data also address a knowledge gap about the establishment post-viral lung pathology, as prior studies employed juvenile-age mice ${ }^{13,14,24,25,27,59,60,61}$. In juvenile mice, induction of chronic mucous cell metaplasia after SeV infection depends on IL-13 production and requires the cooperation of two groups of cells: 1) IL-33 producing epithelial cells (type-ll pneumocytes and a subset of airway progenitor cells) that proliferate in response to acute viral infection as part of the repair process; and 2) a collection of innate immune cells (iNKTs, tissue macrophages, and ILC2s) responsible for generating IL-13 in response to IL-33 stimulation $14,24,25,59,61$. Although the induction of post-viral lung pathology by SeV only becomes histologically evident by $21 \mathrm{DPI}^{13}$, we observed a much earlier divergence between juvenile and mature 
mice at $5 \mathrm{DPI}$ in terms of airway hyper-responsiveness, type 2 cytokine production, and $I L-33$ induction during acute $\mathrm{SeV}$ infection. Our data suggest that early host responses during acute viral illness can be decisive for the later establishment of asthmatic phenotypes, and these responses are at least in part modified by developmental aging.

Our data also suggest a role for the lung immune micro-environment and particularly tissue-resident AMs in the aging out of post-viral lung pathology. AMs represent the major immune cell in the lung microenvironment at baseline, and are a major source of IFNa/ $\beta$ production early in RNA respiratory viral infections ${ }^{62}$. Studies show that AM depletion modifies the severity of various acute viral infections, in some cases exacerbating illness (influenza, RSV, vaccinia, and SeV as shown here) ${ }^{63,64,65}$, and in other cases reducing acute illness (MHV-1 and mouse adapted SARS-CoV-1) ${ }^{66,67}$. Interestingly, studies show that AM depletion exacerbates type 2 airway inflammation and mucous cell metaplasia immediately after allergen exposure, although the duration of these effects were not evaluated ${ }^{68,69}$. Thus, AMs would seem a logical cell to influence acute host responses to viruses that later modulate chronic asthma phenotypes. Consistent with prior reports ${ }^{39}$, we observed reduced AM abundance and proliferative capacity in older mice. However, our data also suggest that AM regulation of post-viral lung pathology is unlikely to be a simple matter of cell number. If it were, juvenile mice that harbor a larger AM population ought to have exhibited milder post-viral phenotypes than mature mice, when in fact the opposite was the case. Moreover, AM depletion would have been predicted to worsen post-viral pathology in juvenile mice, but the effects were instead seen in mature mice whose macrophage abundance is less affected by clodronate treatment. Rather, our data suggest that aging of the lung micro-environment, as reflected by AM immunophenotype and differential gene expression, drive the attenuation of post-viral asthma phenotypes in adult mice. Such an interpretation is based on studies demonstrating the strong inductive effect of micro-environment on gene expression in tissue resident macrophages, including $\mathrm{AMs}^{70,71}$. The extent to which age-related changes to AM programming reflect cell-intrinsic timekeeping ${ }^{72,73}$ or structural modifications to the lung microenvironment over time ${ }^{74}$ is an important question for future research.

Using unsupervised mass cytometric and transcriptomic approaches, we identified upregulation of MHC-II pathway genes as a major informatic signature of AM aging. The functional significance of this change to AMs is at present unclear and will be the subject of future research. Our observation is compatible with prior reports of MHC-II upregulation in AMs from aged mice (> 22 months old), particularly on CD11 $\mathrm{b}^{+}$ $\mathrm{AMs}^{75}$. Because MHC-III AMs displayed more efficient phagocytosis of Mycobacterium tuberculosis (MTb) bacteria, the authors suggested senile AM changes could help explain why elderly patients are more susceptible to $\mathrm{MTb}^{75}$. However, our study adds a key observation: MHC-II upregulation on AMs is saturating by 7 months of age in healthy mice and therefore correlates with the completion of adult maturation, not old age. The distinction is significant because published studies involving aging and immunologic responses usually report a comparison of extremes between "old" (generally > 18 months old) and "young" animals (variably defined as 4 weeks to 4 months old). However, aging occurs continuously across the lifespan of an organism and there may be significant milestones relevant to disease between the extremes of age, milestones that could be missed or misinterpreted by a dichotomous approach to this biological 
variable. The phenomenon of asthma remission makes clear that clinically important changes to host responses occur across the childhood to adulthood aging landscape, and we show some of these can be recapitulated in mouse models. Research is needed to more precisely map how host-responses evolve across the entire continuum of aging.

In summary, we report an attenuation of post-viral chronic lung pathology and asthmatic phenotypes with maturation in mice. Alveolar macrophages have a role in mediating these age-related inflammatory differences. Our findings have implications for the biological basis of asthma remission in children.

\section{Materials And Methods}

Mice. C57BL/6J mice (4-8 weeks old, 20-25g) were sourced from Jackson Laboratories and acclimatized at least one week before use. We employed a range of ages spanning 5 weeks to 18 months for experiments. We adhered to the Jackson Laboratories convention of defining "juvenile" as 3 months or less and "mature" as greater than six months ${ }^{15}$. In some experiments, older mice (>9 months) were sourced from retired C57BL/6J breeders obtained from the Washington University School of Medicine Microinjection Core/Gnotobiotic Facility. Unless stated, male mice were used for experiments. Mice were housed in a standard 12-hour light-dark cycle (lights-on at 6:00 AM and lights-off at 6:00 PM local time), with unlimited food and water available. For certain experiments (Supplementary Fig. 3), mice were fed either a high fat $(60 \%)$ or low fat (10\%) diet (Envigo) prior to viral infection. Otherwise, they received $20 \%$ fat content chow, which is the standard at our animal facility. All experiments were approved by the Washington University School of Medicine Animal Care and Use Committees.

Microbiome depletion. Mice were treated with broad-spectrum antibiotics to deplete and normalize their microbiomes as described ${ }^{23}$. Briefly, three weeks before $\mathrm{SeV}$ infection, water provided to mice was replaced for two weeks by either control sugar solution $\left(20 \mathrm{~g} / \mathrm{L} \mathrm{grape} \mathrm{Kool-Aid}^{\mathrm{TM}}\right)$ or high dose VNAM solution (sugar solution plus $0.5 \mathrm{~g} / \mathrm{L}$ vancomycin, $1 \mathrm{~g} / \mathrm{L}$ neomycin, $1 \mathrm{~g} / \mathrm{L}$ ampicillin, and $1 \mathrm{~g} / \mathrm{L}$ metronidazole (SigmaAldrich)). Then, for the week prior to and one week after SeV infection mice received a low dose VNAM solution ( $25 \mathrm{~g} / \mathrm{L}$ sugar solution plus $0.35 \mathrm{~g} / \mathrm{L}$ vancomycin, and $0.75 \mathrm{~g} / \mathrm{L}$ metronidazole (Sigma-Aldrich)) or control sugar solution. Stool samples were obtained prior to VNAM treatment, on the day of virus infection, and on the day VNAM treatment ended. To confirm microbiome depletion, stool DNA was extracted from fresh samples using a commercial kit (Qiagen), and DNA content per mg stool was determined using a Nanodrop 2000 spectrophotometer (Thermo).

Microbiome analysis. Stool DNA obtained from juvenile and mature mice was extracted using a commercial kit (Qiagen). Normalized fecal DNA obtained from juvenile and mature mice was subjected to V4-16S rRNA PCR as previously described ${ }^{76}$ using custom Illumina adapted primers. Briefly, these primers were designed to support combinatorial based demultiplexing of samples based on their position within a 96 well plate. The forward primer contains a $5^{\prime}$ Illumina specific i7 sequence, followed by a plate specific barcode (italics), a primer pad site, a linker sequence, a row-specific sequence (underlined) ${ }^{77}$, then the V4$16 \mathrm{~S} 515 \mathrm{~F}$ sequence ${ }^{78}$ on the $3^{\prime}$ end: 

XXXXXXXX-GTGYCAGCMGCCGCGGTAA-3'.

The reverse primer contains a 5' Illumina i5 sequence, followed by a primer pad site, a linker sequence, a column-specific sequence (underlined), then a 3' V4-16S 806R sequence ${ }^{79}$ :

\section{5'-AATGATACGGCGACCACCGAGATCTACAC-TCTTTCCCTACACGACGCTCTTCCGATCT-GT-XXXXXXXX- GGACTACNVGGGTWTCTAAT-3'.}

Following PCR, V4-16S amplicons were quantitated, pooled in an equimolar ratio, then purified (Ampure XP beads; Beckman Coulter) before sequencing on an Illumina MiSeq instrument using 2 x 250 base pair chemistry. Following demultiplexing, reads were trimmed and then Amplicon sequence variants (ASVs) were generated using the large-data adapted pipeline for DADA2 version 1.10.1 in $\mathrm{R}^{80}$. Sequences were classified to the highest taxonomic resolution possible using the RDPClassifier and a minimum bootstrap support of $80 \%{ }^{81}$. Analysis of ASV abundance was performed in R version 3.5.3 using phyloseq version $1.26 .1^{82}$. Bray-Curtis distances were used to perform principal coordinates analysis on mouse microbiota over the treatment interval.

Virus infections. Sendai virus (SeV) was obtained from ATCC (VR-105; Sendai/52). Virus was twice plaque purified, propagated in 10-day-old embryonated chicken eggs, and stored at -70C. Infectivity of SeV was assayed in VeroE6 cell line as described ${ }^{33}$. Influenza A virus (IAV A/WS/33 H1N1) was obtained from ATCC. Virus was plaque purified using MDCK (ATCC CCL-34) cells and propagated in 9-day-old embryonated chicken eggs. SeV at various doses or 5 pfu of H1N1 influenza A virus was intranasally administered to mice as described ${ }^{16,33}$. Briefly, animals were anesthetized with isoflurane, and virus was intranasally instilled in $30 \mu$ l of PBS. Mice were then returned to their cages to recover from anesthesia.

Ovalbumin (Ova) sensitization. Ova airway sensitization was performed as a 21-day protocol essentially as described $^{18}$. Briefly, mice were injected i.p. with $0.4 \mathrm{ml}$ of an $0 v a /$ Alum slurry on days 0 and 7 (the slurry contained $0.42 \mathrm{mg}$ Ova (Fraction V, Sigma) and 1.9mg Alum-Inject (Pierce) diluted in PBS). On day 21, mice were anesthetized using ketamine/xylazine, and Ova was intra-nasally instilled (200 $\mu \mathrm{g}$ Ova in 50 $\mu$ of PBS). Two days after intranasal challenge, mice were euthanized, and lungs were obtained for gene expression analysis.

Alternaria sensitization. Mice were sensitized to Alternaria Alternata extract (Fisher Scientific) essentially as described $^{83}$. Briefly, mice were anesthetized with $2 \%$ isoflurane, and Alternaria extract $(25 \mu \mathrm{g}$ in $25 \mu \mathrm{l}$ sterile PBS) was instilled intranasally. Mice received a total of 5 doses of Alternaria extract every 2 days and then were euthanized for analysis 12 days after the initial dose.

AM depletion. To deplete AMs, mice were intranasally treated with clodronate liposomes like prior reports ${ }^{41}$. Briefly, isoflurane-anesthetized mice were intranasally administered $40 \mu$ high potency clodronate liposomes $(20 \mathrm{mg} / \mathrm{mL}$ ) or control liposomes (FormuMax) 4 days and 2 days prior to SeV infection. 
RNA extraction and quantitative PCR (qPCR). At indicated times after infection, lungs were manually excised and immersed in RNAlater solution (Applied Biosystems). Total RNA was isolated from lung homogenates using RNeasy Mini Kits (Qiagen). Reverse transcription was carried out using the Applied Biosystems High Capacity cDNA Reverse Transcription Kit. Real Time qPCR was performed on the ABI 7500 Fast Real-Time PCR system. Primers used for qPCR are listed in Supplementary Table 1.

Bulk RNA Sequencing. Bulk RNAseq was performed at the Washington University Genome Technology Access Center (GTAC) using standard protocols. Briefly, RNA from whole lung homogenates were converted to cDNA libraries for sequencing using the RiboZero Kit (Sigma Aldrich). Libraries generated from BAL RNA were generated using the SMARTer kit (Clontech). Sequencing was carried out on a NovaSeq6000 instrument (Illumina). RNA-seq reads were aligned to the Ensembl release 76 assembly with STAR version 2.0.4b. Gene counts were derived from the number of uniquely aligned unambiguous reads by Subread:featureCount version 1.4.5. Transcript counts were produced by Sailfish version 0.6.3. Sequencing performance was assessed for total number of aligned reads, total number of uniquely aligned reads, genes and transcripts detected, ribosomal fraction known junction saturation, and read distribution over known gene models with RSeQC version 2.3. All gene-level and transcript counts were then imported into the R/Bioconductor package EdgeR and TMM normalization size factors were calculated to adjust for samples for differences in library size. Genes or transcripts not expressed in any sample were excluded from further analysis. The TMM size factors and the matrix of counts were imported into R/Bioconductor package Limma ${ }^{84}$, and weighted likelihoods based on the observed mean-variance relationship of every gene/transcript were then calculated for all samples with the Voom function ${ }^{85}$. Performance of the samples was assessed with a Spearman correlation matrix and multi-dimensional scaling plots. Gene/transcript performance was assessed with plots of residual standard deviation of every gene to their average log-count with a fitted trend line of the residuals. Generalized linear models with dispersion estimates were then created to test for gene/transcript level differential expression. Differentially expressed genes and transcripts were then filtered for FDR adjusted p-values less than or equal to 0.05 . To examine age-associated differential gene expression in BAL samples, Log $_{2}$ normalized Voom-adjusted CPM data was analyzed for statistical significance by the Kruskal-Wallis one-way analysis of variance by ranks.

Single Cell RNA Sequencing (scRNAseq) of BAL cells. Pooled BAL cell samples from healthy juvenile (6 weeks-old, $n=10$ ) and mature (12 months old, $n=9$ ) mice were subjected to scRNAseq at the Washington University Genome Technology Access Center (GTAC) using standard protocols. Briefly, single cell barcoded cDNA libraries were generated using a Chromium microfluidics platform (10x Genomics) and the Chromium Single Cell $3 \bowtie$ GEM, Library \& Gel Bead Kit v3 (10x Genomics). Sequencing was carried out on a NovaSeq6000 instrument (Illumina). Sequencing data was processed using Cell Ranger version 3.1, starting with demultiplexed FASTQ files and using GRCm38-2.0.1 as the reference genome. Processed scRNAseq data was analyzed using $R$ version 3.6.2. and the SEURAT v3 package as described ${ }^{86}$ (available at https://satijalab.org/seurat/). We used previously published marker genes to type cell clusters identified on UMAP analysis (see Supplementary Table 2) ${ }^{87,88}$. 
Morphometry. Lungs were fixed by inflation with $10 \%$ buffered formalin under $20 \mathrm{cmH}_{2} \mathrm{O}$ of pressure, paraffin imbedded, and stained with PAS or subjected to immunofluorescence (IF) staining using standard techniques ${ }^{33}$. PAS-stained slides were scanned using a Hamamatsu Nanozoomer at the Alafi Neuroimaging lab at Washington University, and PAS positive pixels were counted using Aperio ScanScope Software as described ${ }^{89}$. For immunofluorescence experiments we utilized antibodies directed against SeV (Abcam, Cat. \#ab33988, 1:500 dilution), Krt17 (Abcam, Cat\# ab53707 diluted 1:100) and acetylated aTubulin (MilliporeSigma, Cat \# T7451 diluted 1:1000), followed by Alexa Fluor-conjugated secondary antibodies and DAPI counterstain (Invitrogen). Stained slides were visualized using an Olympus BX51 microscope and a Retiga-2000R camera system (QImaging). We used a double-blind design to quantitate $\mathrm{SeV}$ infection in lung histological sections. After masking sample identification, 5 microscopic fields were randomly selected at $10 \mathrm{X}$ magnification per lung section. $\mathrm{SeV}^{+}$and total cell counts were quantified using ImageJ software.

Broncheoalveolar Lavage (BAL) and Cytokine Analysis. BAL analysis was performed as described ${ }^{33}$. Briefly mice were euthanized, and their tracheas were cannulated with a 20G flexible catheter, 1-2 $\mathrm{mm}$ distal to the larynx. The lungs were then slowly lavaged in 2 passes with a total of $1 \mathrm{ml}$ sterile phosphate buffered saline (PBS). For BAL cell counts, $50 \mu \mathrm{l}$ of BAL suspension was adsorbed to frosted glass slides using a Cytospin 4 centrifuge (Thermo Fischer) and treated with Diff-Kwik stain (Thermo Fischer). Slides were then digitally scanned at 20X magnification using a Nanozoomer HT imaging system (Hamamatsu). To quantify cell number, scanned slides were analyzed via ImageScope software (Leica) using the "Nuclear v9" algorithm, which counts nuclei in histologically stained samples. For BAL cell differential, sample identifications were blinded and 5 random 40x fields were captured as TIFF files using ImageScope software. Cells in each field were then scored as either lymphocytes, macrophages, or neutrophils by a separate investigator also blinded to the sample ID. A minimum of 50 cells were counted per biological specimen. BAL protein concentration was quantified using Bradford reagent (Sigma) according to standard protocols. BAL cytokine levels were analyzed at the Washington University Immunomonitoring Core Facility on a FLEXMAP 3D instrument (Luminex) and using the Mouse Cytokine and Chemokine Panel 1A (Affymetrix), according to the manufacturer's instructions.

Lung Mechanics Measurement. Airway resistance was measured using a Buxco Elan RC mouse volumecontrolled ventilator as described ${ }^{33}$. Briefly, mice were anesthetized by i.p. injection of ketamine/xylazine cocktail, then tracheostomy was performed using a 22-gauge flexible catheter. Mice were then ventilated and given progressively doubled concentrations of methacholine through an in-line nebulizer. Inspiratory resistance measurements were averaged over three-minute intervals after each dose.

Mass and Flow Cytometry. Single lung cell suspensions were prepared using Liberase-TM and DNase in RPMI (Roche) essentially as described ${ }^{33}$. Cells were stained using panels of metal-conjugated antibodies or fluorophore-conjugated antibodies for mass and flow cytometry, respectively (Supplementary Tables 3 and 4). Mass Cytometry was carried out at the Washington University Immunomonitoring Laboratory using a CyTOF2/Helios instrument (Fluidigm) and analyzed using CytoBank software as described ${ }^{90}$. Flow cytometry was performed at the Washington University Siteman Flow Cytometry Core using an Attune NxT 
instrument (ThermoFischer) and was analyzed using FlowJo software (BD Biosciences). See Supplementary Fig. 6a and 7a for representative gating strategies for mass and flow cytometry, respectively. For flow cytometry 50,000 events per sample were targeted.

DQ-Ova assay. DQ-Ova assay was conducted similarly to prior reports ${ }^{37}$. Isoflurane-anesthetized mice were intranasally treated with $30 \mu \mathrm{g}$ DQ-Ova (Thermo Fischer) in $30 \mu \mathrm{l}$ of sterile PBS. Two hours later, mice were euthanized, and flow cytometry of whole lung cell suspensions was conducted as above using an antibody cocktail (Supplementary Table 4). A representative gating strategy is presented in Supplementary Fig. 7b.

Statistics. For statistical testing of flow-cytometric, qPCR, and BAL cytokine data, Student's T-tests were performed on log-normalized data as recommended ${ }^{91}$ using Microsoft Excel. For survival curve analysis the log-rank test was used. UMAP analysis was performed using the SEURAT package in R as described above. viSNE analysis of mass cytometry data was performed using CytoBank software. Principal component analysis of BAL cytokines and mass cytometry data was performed in Microsoft Excel using a freeware add-in (available at http://wak2.web.rice.edu/bio/Kamakura_Analytic_Tools.html). KEGG pathway functional enrichment analysis was conducted using DAIVD v6.8 software ${ }^{92,93}$ (available at https://david.ncifcrf.gov/home.jsp). All morphometric analyses were double blinded.

Methodological Limitations. It is worth mentioning some limitations to our methodological approach. Although mice are frequently used as a model organism to study inflammatory airway pathology, how far results obtained from mouse models can be generalized to human asthma is controversial ${ }^{94}$. The scope of our data is most applicable to type $2^{\mathrm{Hi}}$ asthmatic endotypes that predominate in children and does not address adult-onset asthma endotypes, which may represent mechanistically distinct entities. Our data examine the pathologic effects of discrete exposures to viruses or allergens, but human asthma involves recurrent exposures to such triggers over time. Further experiments involving recurrent viral challenge at different stages of development and correlation with biomarker studies in human asthma remission cohorts represent possible next steps. All considered however, our approach supplies a needed mouse model that can recapitulate aspects of childhood asthma remission. We anticipate it will facilitate future investigation of this poorly understood phenomenon.

Resource Availability. This study did not generate new reagents. Bulk and single cell RNA seq data (Figs. 4, $\mathbf{8}$, and $\mathbf{9}$ ) is being uploaded to GEO and will be complete prior to publication of this manuscript. R code for analyzing single cell RNAseq data in SEURAT can be found at the following link:

https://www.dropbox.com/s/ovikd97763kn1y5/Hazan\%20et\%20al_ScRNA\%20analysis\%20R\%20code.pdf? $\mathrm{dl}=0$

Online supplemental material. Supplementary Fig. 1 is associated with Fig. 1 and compares SeV induction of MUC5AC expression in male and female mice of different ages at 49 DPI. Supplementary Fig. 2 is associated with Fig. 1 and compares the effect of Alternaria alternata extract on lung pathology in juvenile and mature mice. Supplementary Fig. $\mathbf{3}$ is associated with Fig. 1 and examines the effect of high fat versus low fat diet on SeV-induced MUC5AC expression in juvenile mice. Supplementary Fig. 4 is associated with 
Fig. 7 and shows lung abundance of selected immunophenotypes after SeV infection using mass cytometry in juvenile and mature mice. Supplementary Fig. $\mathbf{5}$ is associated with Fig. 10 and provides additional data on the effects of intranasal clodronate treatment or control liposome treatment in mice.

Supplementary Fig. 6 is associated with Figs. 7 and Supplementary 4 and provides the gating strategy used in this paper for mass cytometry analysis. Supplementary Fig. $\mathbf{7}$ is associated with Fig. $\mathbf{8}$ and provides the gating strategy for color flow cytometry and the DQ-Ova assay. Supplementary Table 1 lists primers used for qPCR throughout the study. Supplementary Table 2 is associated with Fig. 9 and lists marker genes used to identify cell types in single cell RNAseq analyses of BAL cells. Supplementary Table $\mathbf{3}$ is associated with Fig. 7 and Supplementary Fig. 4 and lists antibodies used for mass cytometry. Supplementary Table 4 is associated with Fig. 8 and lists antibodies used for color flow cytometry.

\section{Declarations}

\section{ACKNOWLEDGEMENTS}

We thank Robyn Puro and Steven Brody for their editorial input. This work was funded by NIH R01 HL135846, R01 HL152968, and a grant from the Children's Discovery Institute.

\section{AUTHOR CONTRIBUTIONS}

$\mathrm{MJH}$ and JH conceived the project. GH, AE, CG, CF, JA, ALK, EA, SPK, JAB, DS, DK, MW, XW, YZ, DB, AHL, ALR, and JH performed experiments. GH, JA, ALK, JRK, and JH analyzed the data. GH, AE, and JH wrote the paper. All authors reviewed the manuscript. JH supervised the study.

\section{COMPETING INTERESTS}

The authors report no competing interests.

\section{References}

1. Holgate, S.T. et al. Asthma. Nat Rev Dis Primers 1, 15025 (2015).

2. Mims, J.W. Asthma: definitions and pathophysiology. Int Forum Allergy Rhinol 5 Suppl 1, S2-6 (2015).

3. Yunginger, J.W. et al. A community-based study of the epidemiology of asthma. Incidence rates, 19641983. The American review of respiratory disease 146, 888-894 (1992).

4. Dharmage, S.C., Perret, J.L. \& Custovic, A. Epidemiology of Asthma in Children and Adults. Front Pediatr 7, 246 (2019).

5. Wenzel, S.E. Asthma phenotypes: the evolution from clinical to molecular approaches. Nature medicine 18, 716-725 (2012).

6. Strachan, D.P., Butland, B.K. \& Anderson, H.R. Incidence and prognosis of asthma and wheezing illness from early childhood to age 33 in a national British cohort. BMJ (Clinical research ed.) 312, 1195-1199 (1996). 
7. Sears, M.R. et al. A longitudinal, population-based, cohort study of childhood asthma followed to adulthood. The New England journal of medicine 349, 1414-1422 (2003).

8. Vonk, J.M. et al. Childhood factors associated with asthma remission after 30 year follow up. Thorax 59, 925-929 (2004).

9. Moorman, J.E. et al. National surveillance of asthma: United States, 2001-2010. Vital Health Stat 3, 158 (2012).

10. Pesce, G. et al. Seventy Years of Asthma in Italy: Age, Period and Cohort Effects on Incidence and Remission of Self-Reported Asthma from 1940 to 2010. PloS one 10, e0138570 (2015).

11. Fleming, D.M., Cross, K.W., Sunderland, R. \& Ross, A.M. Comparison of the seasonal patterns of asthma identified in general practitioner episodes, hospital admissions, and deaths. Thorax 55, 662-665 (2000).

12. Sears, M.R. Epidemiology of asthma exacerbations. The Journal of allergy and clinical immunology 122, 662-668 (2008).

13. Walter, M.J., Morton, J.D., Kajiwara, N., Agapov, E. \& Holtzman, M.J. Viral induction of a chronic asthma phenotype and genetic segregation from the acute response. The Journal of clinical investigation 110, 165-175 (2002).

14. Byers, D.E. et al. Long-term IL-33-producing epithelial progenitor cells in chronic obstructive lung disease. The Journal of clinical investigation 123, 3967-3982 (2013).

15. Flurkey, K. \& Currer, J. The Jackson Laboratory Handbook on Genetically Standardized Mice Sixth Edition, 6th edn. The Jackson Laboratory: Bar Harbor, ME 04609 USA, 2009.

16. Keeler, S.P. et al. Influenza A Virus Infection Causes Chronic Lung Disease Linked to Sites of Active Viral RNA Remnants. Journal of immunology (Baltimore, Md. : 1950) 201, 2354-2368 (2018).

17. Rosenberg, H.F. \& Druey, K.M. Modeling asthma: Pitfalls, promises, and the road ahead. J Leukoc Biol 104, 41-48 (2018).

18. Busse, P.J., Zhang, T.F., Srivastava, K., Schofield, B. \& Li, X.M. Effect of ageing on pulmonary inflammation, airway hyperresponsiveness and $T$ and $B$ cell responses in antigen-sensitized and challenged mice. Clinical and experimental allergy : journal of the British Society for Allergy and Clinical Immunology 37, 1392-1403 (2007).

19. Bartemes, K.R. et al. IL-33-Responsive Lineage<sup $>-</$ sup $>$ CD $25<$ sup $>+</$ sup $>C D 44<$ sup $>$ hi $</$ sup $>$ Lymphoid Cells Mediate Innate Type 2 Immunity and Allergic Inflammation in the Lungs. The Journal of Immunology 188, 1503-1513 (2012).

20. Reynolds, T.H. et al. The impact of age and sex on body composition and glucose sensitivity in C57BL/6J mice. Physiological reports 7, e13995 (2019).

21. Langille, M.G.I. et al. Microbial shifts in the aging mouse gut. Microbiome 2, 50 (2014).

22. Duan, Y. et al. Inflammatory Links Between High Fat Diets and Diseases. Frontiers in immunology 9 , 2649 (2018).

23. Steed, A.L. et al. The microbial metabolite desaminotyrosine protects from influenza through type I interferon. Science (New York, N.Y.) 357, 498-502 (2017). 
24. Kim, E.Y. et al. Persistent activation of an innate immune response translates respiratory viral infection into chronic lung disease. Nature medicine 14, 633-640 (2008).

25. Wu, K. et al. TREM-2 promotes macrophage survival and lung disease after respiratory viral infection. The Journal of experimental medicine 212, 681-697 (2015).

26. Pichery, M. et al. Endogenous IL-33 is highly expressed in mouse epithelial barrier tissues, lymphoid organs, brain, embryos, and inflamed tissues: in situ analysis using a novel II-33-LacZ gene trap reporter strain. Journal of immunology (Baltimore, Md. : 1950) 188, 3488-3495 (2012).

27. Wang, X. et al. TLR3-Activated Monocyte-Derived Dendritic Cells Trigger Progression from Acute Viral Infection to Chronic Disease in the Lung. Journal of immunology (Baltimore, Md. : 1950) (2021).

28. DePianto, D., Kerns, M.L., Dlugosz, A.A. \& Coulombe, P.A. Keratin 17 promotes epithelial proliferation and tumor growth by polarizing the immune response in skin. Nature Genetics 42, 910-914 (2010).

29. Zhang, X., Yin, M. \& Zhang, L.J. Keratin 6, 16 and 17-Critical Barrier Alarmin Molecules in Skin Wounds and Psoriasis. Cells 8 (2019).

30. Look, D.C. et al. Effects of paramyxoviral infection on airway epithelial cell Foxj1 expression, ciliogenesis, and mucociliary function. Am J Pathol 159, 2055-2069 (2001).

31. Hussell, T. \& Bell, T.J. Alveolar macrophages: plasticity in a tissue-specific context. Nat Rev Immunol 14, 81-93 (2014).

32. Lavin, Y. et al. Tissue-Resident Macrophage Enhancer Landscapes Are Shaped by the Local Microenvironment. Cell 159, 1312-1326 (2014).

33. Ehlers, A. et al. BMAL1 links the circadian clock to viral airway pathology and asthma phenotypes. Mucosal immunology 11, 97-111 (2018).

34. Califano, D., Furuya, Y. \& Metzger, D.W. Effects of Influenza on Alveolar Macrophage Viability Are Dependent on Mouse Genetic Strain. Journal of immunology (Baltimore, Md. : 1950) 201, 134-144 (2018).

35. Amir el, A.D. et al. viSNE enables visualization of high dimensional single-cell data and reveals phenotypic heterogeneity of leukemia. Nat Biotechno/ 31, 545-552 (2013).

36. Misharin, A.V., Morales-Nebreda, L., Mutlu, G.M., Budinger, G.R. \& Perlman, H. Flow cytometric analysis of macrophages and dendritic cell subsets in the mouse lung. American journal of respiratory cell and molecular biology 49, 503-510 (2013).

37. von Garnier, C. et al. Allergic airways disease develops after an increase in allergen capture and processing in the airway mucosa. Journal of immunology (Baltimore, Md. : 1950) 179, 5748-5759 (2007).

38. Reith, W., LeibundGut-Landmann, S. \& Waldburger, J.M. Regulation of MHC class II gene expression by the class II transactivator. Nat Rev Immuno/ 5, 793-806 (2005).

39. Wong, C.K. et al. Aging Impairs Alveolar Macrophage Phagocytosis and Increases Influenza-Induced Mortality in Mice. Journal of immunology (Baltimore, Md. : 1950) 199, 1060-1068 (2017).

40. McQuattie-Pimentel, A.C. et al. The lung microenvironment shapes a dysfunctional response of alveolar macrophages in aging. The Journal of clinical investigation 131 (2021). 
41. Gibbings, S.L. et al. Transcriptome analysis highlights the conserved difference between embryonic and postnatal-derived alveolar macrophages. Blood 126, 1357-1366 (2015).

42. Feuerstein, J.D. et al. AGA Clinical Practice Guidelines on the Management of Moderate to Severe Ulcerative Colitis. Gastroenterology 158, 1450-1461 (2020).

43. Fanouriakis, A. et al. 2019 update of the EULAR recommendations for the management of systemic lupus erythematosus. Ann Rheum Dis 78, 736-745 (2019).

44. Lichtenstein, G.R. et al. ACG Clinical Guideline: Management of Crohn's Disease in Adults. Am J Gastroentero/ 113, 481-517 (2018).

45. Mian, A., Ibrahim, F. \& Scott, D.L. A systematic review of guidelines for managing rheumatoid arthritis. BMC Rheumato/ 3, 42 (2019).

46. 2020., G.I.f.A.G.S.f.A.M.a.P. Global Initiative for Asthma. Global Strategy for Asthma Management and Prevention 2020., 2020.

47. Expert Panel Working Group of the National Heart, L. et al. 2020 Focused Updates to the Asthma Management Guidelines: A Report from the National Asthma Education and Prevention Program Coordinating Committee Expert Panel Working Group. The Journal of allergy and clinical immunology $146,1217-1270$ (2020).

48. Carpaij, O.A. et al. Childhood factors associated with complete and clinical asthma remission at 25 and 49 years. The European respiratory journal 49 (2017).

49. van den Toorn, L.M., Overbeek, S.E., Prins, J.B., Hoogsteden, H.C. \& de Jongste, J.C. Asthma remission: does it exist? Current opinion in pulmonary medicine 9, 15-20 (2003).

50. Jartti, T., Bonnelykke, K., Elenius, V. \& Feleszko, W. Role of viruses in asthma. Semin Immunopathol 42, 61-74 (2020).

51. De Marco, R. et al. Incidence and remission of asthma: a retrospective study on the natural history of asthma in Italy. The Journal of allergy and clinical immunology 110, 228-235 (2002).

52. Johnston, N.W. et al. The September epidemic of asthma exacerbations in children: a search for etiology. The Journal of allergy and clinical immunology 115, 132-138 (2005).

53. Adeli, M., El-Shareif, T. \& Hendaus, M.A. Asthma exacerbation related to viral infections: An up to date summary. J Family Med Prim Care 8, 2753-2759 (2019).

54. Heymann, P.W. et al. Viral infections in relation to age, atopy, and season of admission among children hospitalized for wheezing. The Journal of allergy and clinical immunology 114, 239-247 (2004).

55. Carpaij, O.A., Burgess, J.K., Kerstjens, H.A.M., Nawijn, M.C. \& van den Berge, M. A review on the pathophysiology of asthma remission. Pharmacol Ther 201, 8-24 (2019).

56. van den Toorn, L.M. et al. Airway inflammation is present during clinical remission of atopic asthma. American journal of respiratory and critical care medicine 164, 2107-2113 (2001).

57. Qi, C. et al. Epigenome-wide association study identifies DNA methylation markers for asthma remission in whole blood and nasal epithelium. Clinical and translational allergy 10, 60 (2020).

58. Vonk, J.M. et al. Novel genes and insights in complete asthma remission: A genome-wide association study on clinical and complete asthma remission. Clinical and experimental allergy : journal of the 
British Society for Allergy and Clinical Immunology 48, 1286-1296 (2018).

59. Alevy, Y.G. et al. IL-13-induced airway mucus production is attenuated by MAPK13 inhibition. The Journal of clinical investigation 122, 4555-4568 (2012).

60. Byers, D.E. et al. Triggering Receptor Expressed on Myeloid Cells-2 Expression Tracks With M2-Like Macrophage Activity and Disease Severity in COPD. Chest 153, 77-86 (2018).

61. Wu, K. et al. Group 2 Innate Lymphoid Cells Must Partner with the Myeloid-Macrophage Lineage for Long-Term Postviral Lung Disease. Journal of immunology (Baltimore, Md. : 1950) 205, 1084-1101 (2020).

62. Kumagai, Y. et al. Alveolar macrophages are the primary interferon-alpha producer in pulmonary infection with RNA viruses. Immunity 27, 240-252 (2007).

63. Schneider, C. et al. Alveolar macrophages are essential for protection from respiratory failure and associated morbidity following influenza virus infection. PLoS Pathog 10, e1004053 (2014).

64. Eichinger, K.M. et al. Alveolar macrophages support interferon gamma-mediated viral clearance in RSVinfected neonatal mice. Respiratory research 16, 122 (2015).

65. Rivera, R. et al. Murine alveolar macrophages limit replication of vaccinia virus. Virology $363,48-58$ (2007).

66. Hartwig, S.M., Holman, K.M. \& Varga, S.M. Depletion of alveolar macrophages ameliorates virusinduced disease following a pulmonary coronavirus infection. PloS one 9, e90720 (2014).

67. Zhao, J., Zhao, J., Van Rooijen, N. \& Perlman, S. Evasion by stealth: inefficient immune activation underlies poor T cell response and severe disease in SARS-CoV-infected mice. PLoS Pathog $\mathbf{5}$, e1000636 (2009).

68. Zasłona, Z. et al. Resident alveolar macrophages suppress, whereas recruited monocytes promote, allergic lung inflammation in murine models of asthma. Journal of immunology (Baltimore, Md. : 1950) 193, 4245-4253 (2014).

69. Mathie, S.A. et al. Alveolar macrophages are sentinels of murine pulmonary homeostasis following inhaled antigen challenge. Allergy 70, 80-89 (2015).

70. Gosselin, D. et al. Environment drives selection and function of enhancers controlling tissue-specific macrophage identities. Cell 159, 1327-1340 (2014).

71. Gautier, E.L. et al. Gene-expression profiles and transcriptional regulatory pathways that underlie the identity and diversity of mouse tissue macrophages. Nat Immunol 13, 1118-1128 (2012).

72. Han, Y. et al. Targeted methods for epigenetic age predictions in mice. Scientific reports 10, 22439 (2020).

73. Levine, M.E. et al. An epigenetic biomarker of aging for lifespan and healthspan. Aging 10, 573-591 (2018).

74. Schulte, H., Mühlfeld, C. \& Brandenberger, C. Age-Related Structural and Functional Changes in the Mouse Lung. Front Physiol 10, 1466 (2019).

75. Lafuse, W.P. et al. Identification of an Increased Alveolar Macrophage Subpopulation in Old Mice That Displays Unique Inflammatory Characteristics and Is Permissive to Mycobacterium tuberculosis 
Infection. Journal of immunology (Baltimore, Md. : 1950) 203, 2252-2264 (2019).

76. Planer, J.D. et al. Development of the gut microbiota and mucosal IgA responses in twins and gnotobiotic mice. Nature 534, 263-266 (2016).

77. Hamady, M., Walker, J.J., Harris, J.K., Gold, N.J. \& Knight, R. Error-correcting barcoded primers for pyrosequencing hundreds of samples in multiplex. Nat Methods 5, 235-237 (2008).

78. Parada, A.E., Needham, D.M. \& Fuhrman, J.A. Every base matters: assessing small subunit rRNA primers for marine microbiomes with mock communities, time series and global field samples. Environ Microbiol 18, 1403-1414 (2016).

79. Apprill, A., McNally, S., Parsons, R. \& Weber, L. Minor revision to V4 region SSU rRNA 806R gene primer greatly increases detection of SAR11 bacterioplankton. Aquatic Microbial Ecology 75, 129-137 (2015).

80. Callahan, B.J. et al. DADA2: High-resolution sample inference from Illumina amplicon data. Nat Methods 13, 581-583 (2016).

81. Wang, Q., Garrity, G.M., Tiedje, J.M. \& Cole, J.R. Naive Bayesian classifier for rapid assignment of rRNA sequences into the new bacterial taxonomy. Appl Environ Microbiol 73, 5261-5267 (2007).

82. McMurdie, P.J. \& Holmes, S. phyloseq: an R package for reproducible interactive analysis and graphics of microbiome census data. PloS one 8, e61217 (2013).

83. Katz-Kiriakos, E. et al. Epithelial IL-33 appropriates exosome trafficking for secretion in chronic airway disease. JCI Insight (2021).

84. Ritchie, M.E. et al. limma powers differential expression analyses for RNA-sequencing and microarray studies. Nucleic Acids Res 43, e47 (2015).

85. Law, C.W., Chen, Y., Shi, W. \& Smyth, G.K. voom: Precision weights unlock linear model analysis tools for RNA-seq read counts. Genome Biol 15, R29 (2014).

86. Stuart, T. et al. Comprehensive Integration of Single-Cell Data. Cell 177, 1888-1902 e1821 (2019).

87. Söllner, J.F. et al. An RNA-Seq atlas of gene expression in mouse and rat normal tissues. Sci Data 4, 170185 (2017).

88. Angelidis, l. et al. An atlas of the aging lung mapped by single cell transcriptomics and deep tissue proteomics. Nature communications 10, 963 (2019).

89. Haspel, J.A. et al. Circadian rhythm reprogramming during lung inflammation. Nature communications 5, 4753 (2014).

90. Brody, S.L. et al. Chemokine Receptor 2-targeted Molecular Imaging in Pulmonary Fibrosis. A Clinical Trial. American journal of respiratory and critical care medicine 203, 78-89 (2021).

91. Genser, B., Cooper, P.J., Yazdanbakhsh, M., Barreto, M.L. \& Rodrigues, L.C. A guide to modern statistical analysis of immunological data. BMC Immuno/ 8, 27 (2007).

92. Huang da, W., Sherman, B.T. \& Lempicki, R.A. Systematic and integrative analysis of large gene lists using DAVID bioinformatics resources. Nat Protoc 4, 44-57 (2009).

93. Huang da, W., Sherman, B.T. \& Lempicki, R.A. Bioinformatics enrichment tools: paths toward the comprehensive functional analysis of large gene lists. Nucleic Acids Res 37, 1-13 (2009). 

respiratory and critical care medicine 174, 1173-1176; discussion 1176-1178 (2006).

\section{Figures}

a

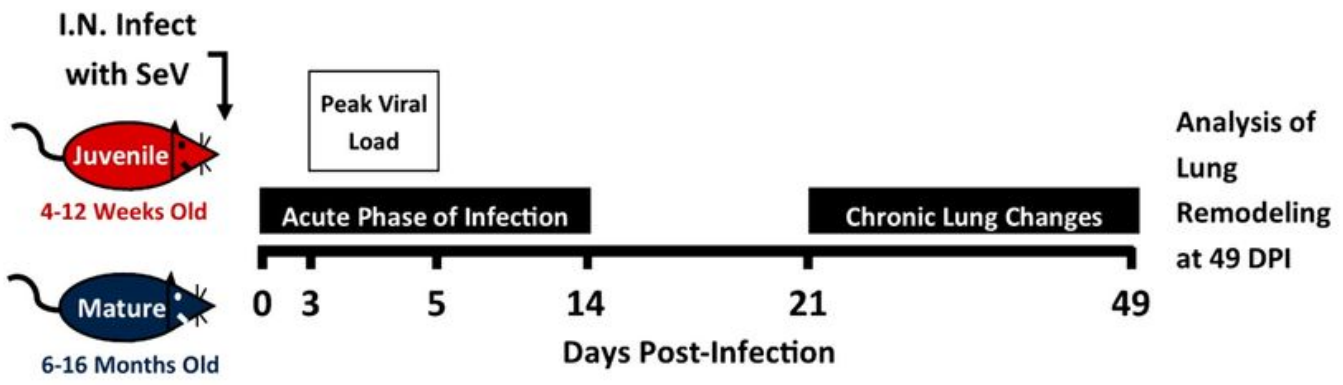

b

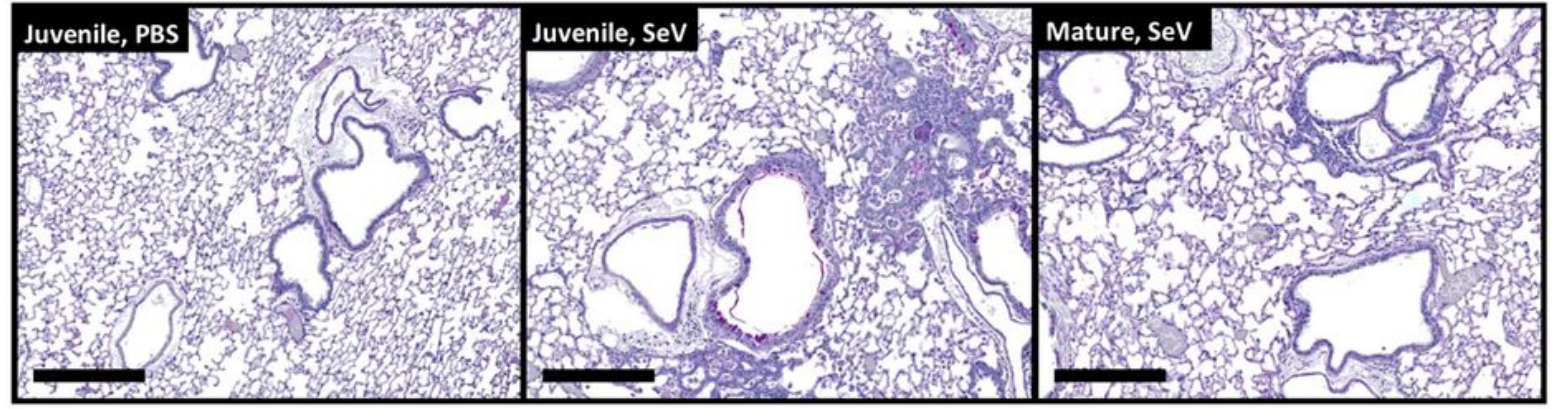

C

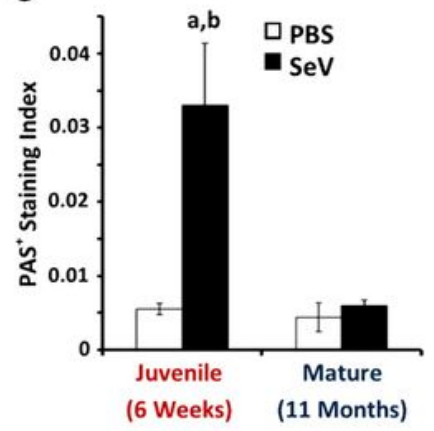

f

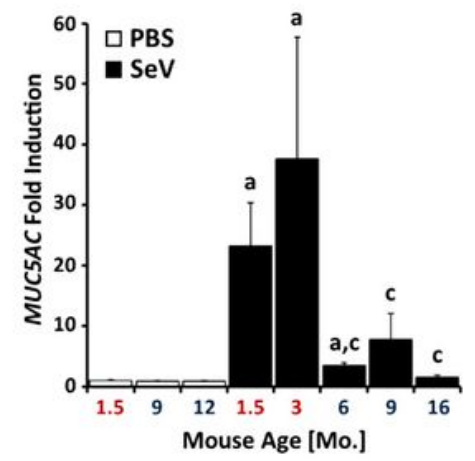

d

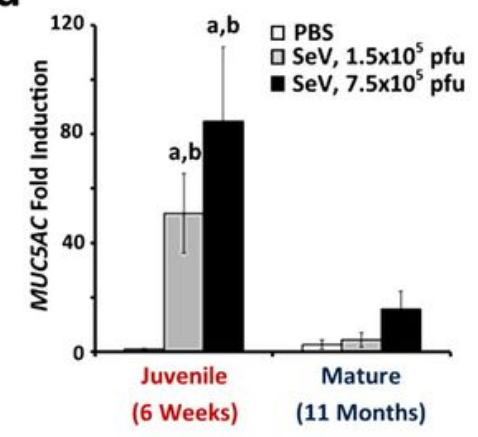

g

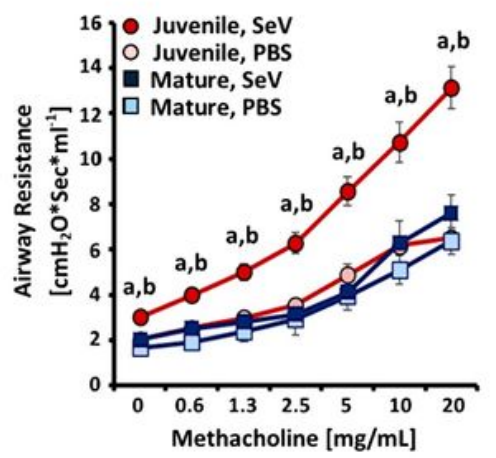

e

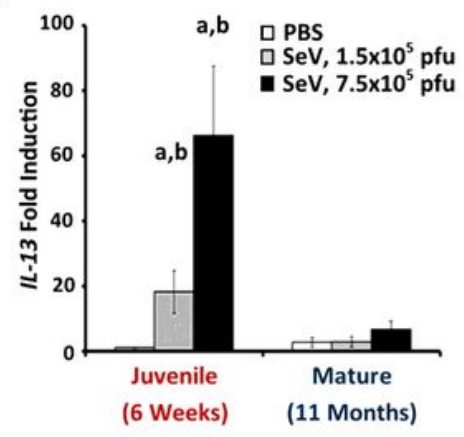

h

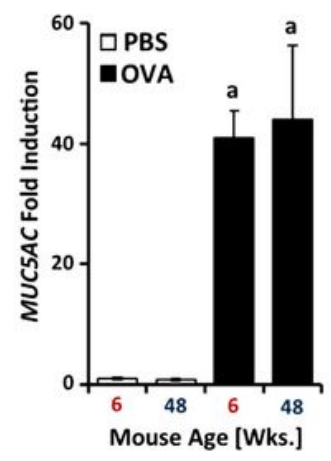

Figure 1 
a

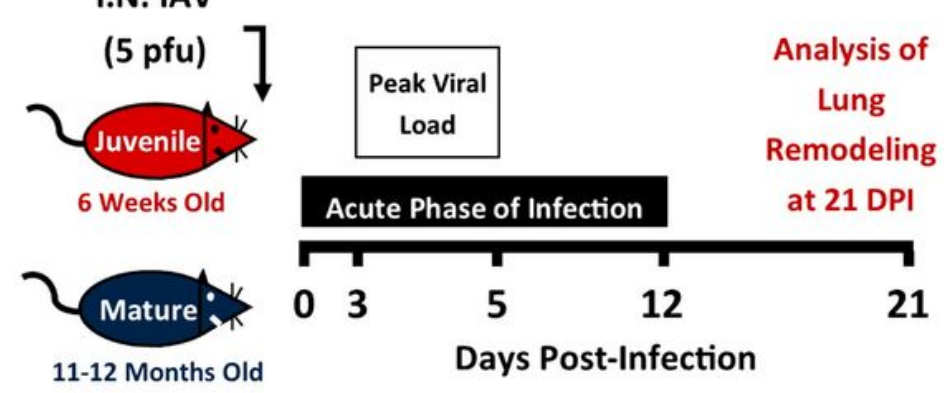

b

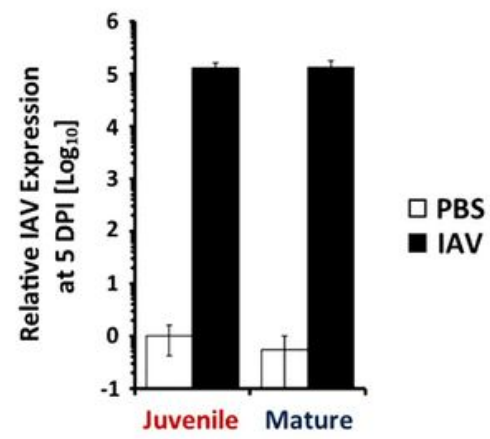

C Juvenile, IAV 21 DPI Mature, IAV 21 DPI

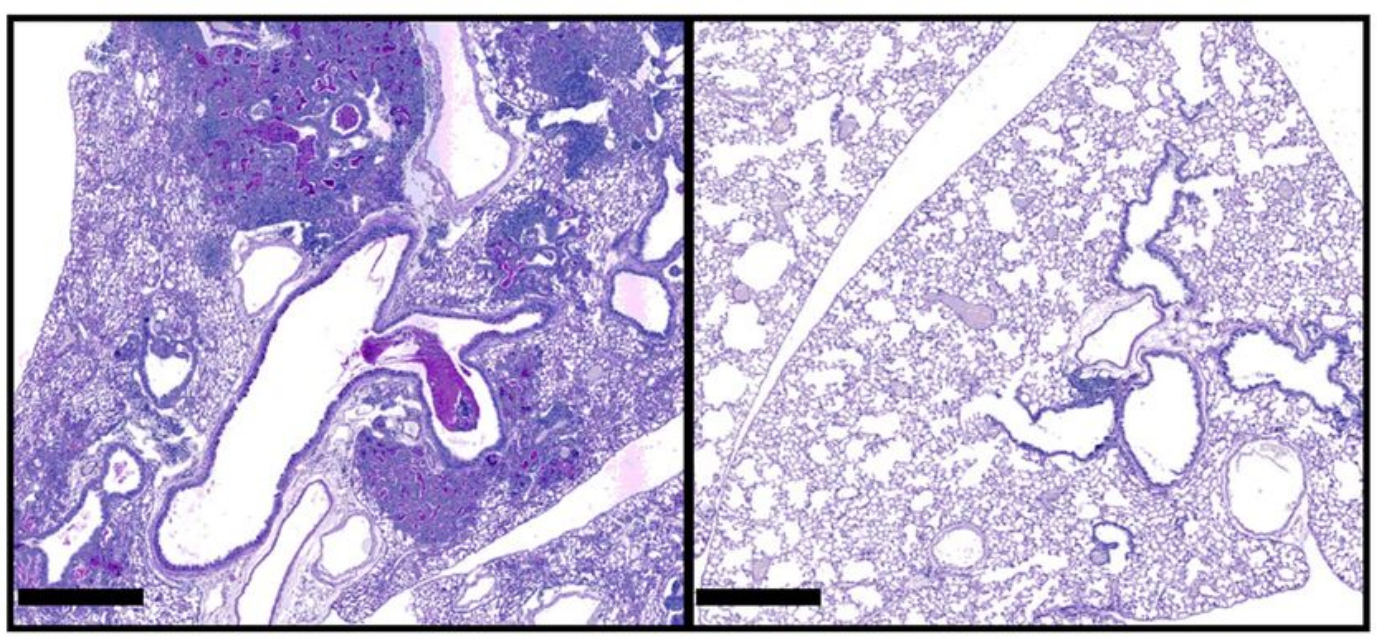

d

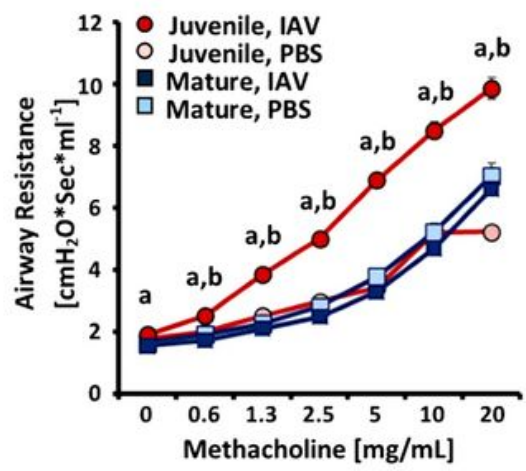

e

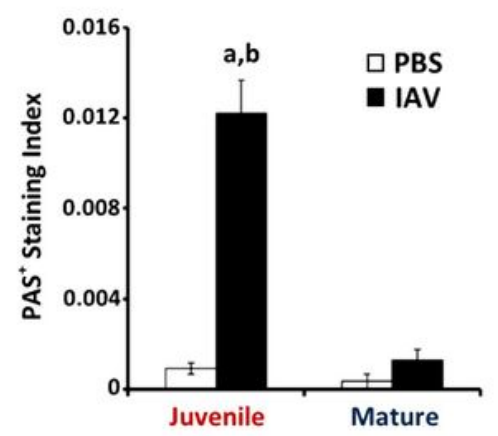

f

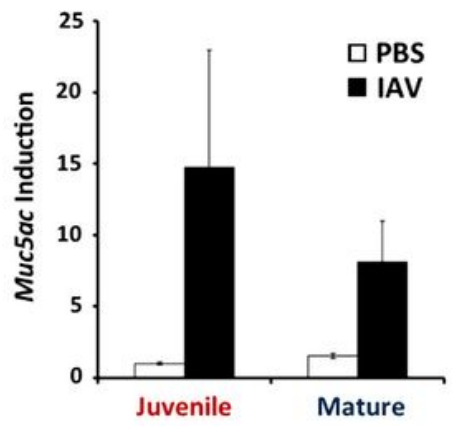

Figure 2

(see Figure Caption file in Supplementary Files) 

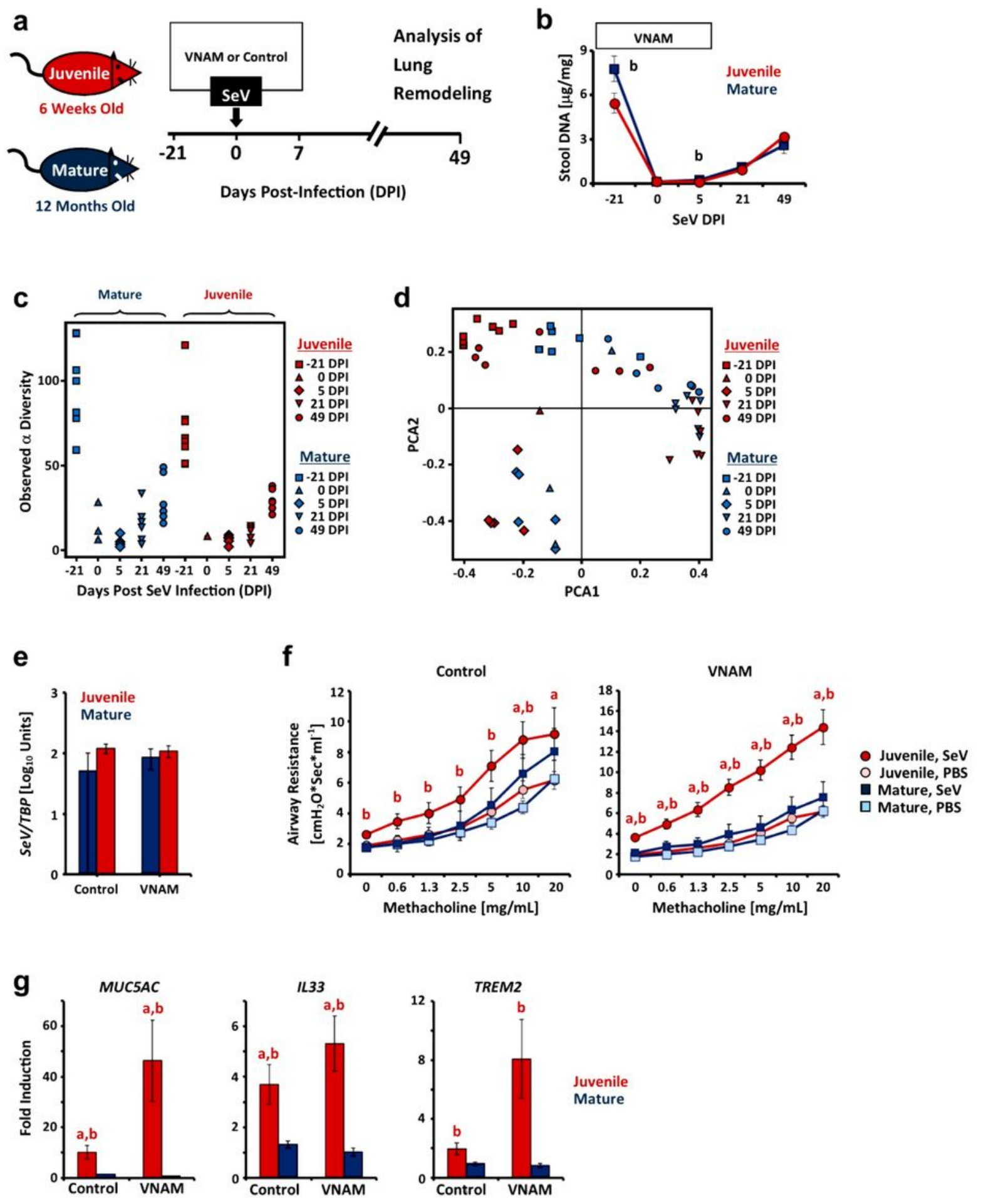

Figure 3

(see Figure Caption file in Supplementary Files) 
a

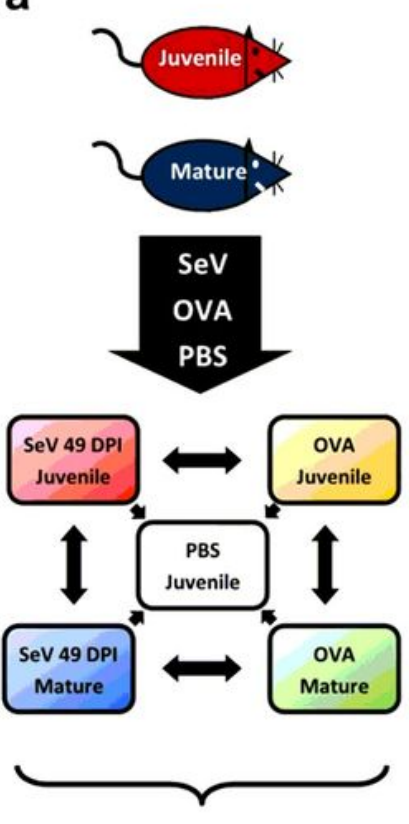

Bulk RNAseq

f

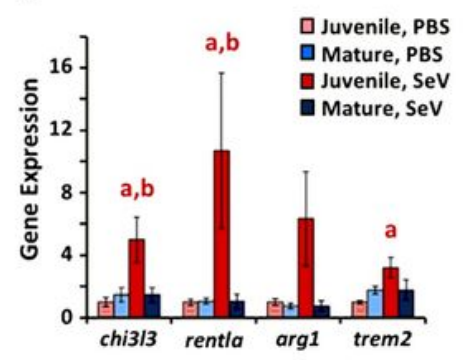

g

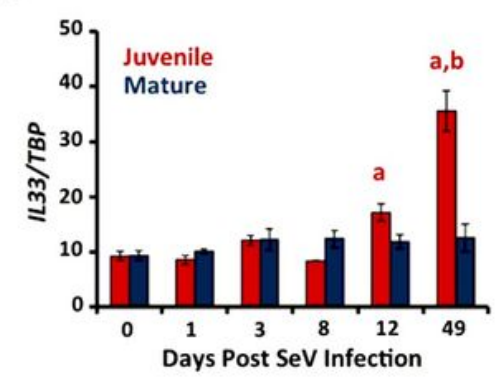

d
C
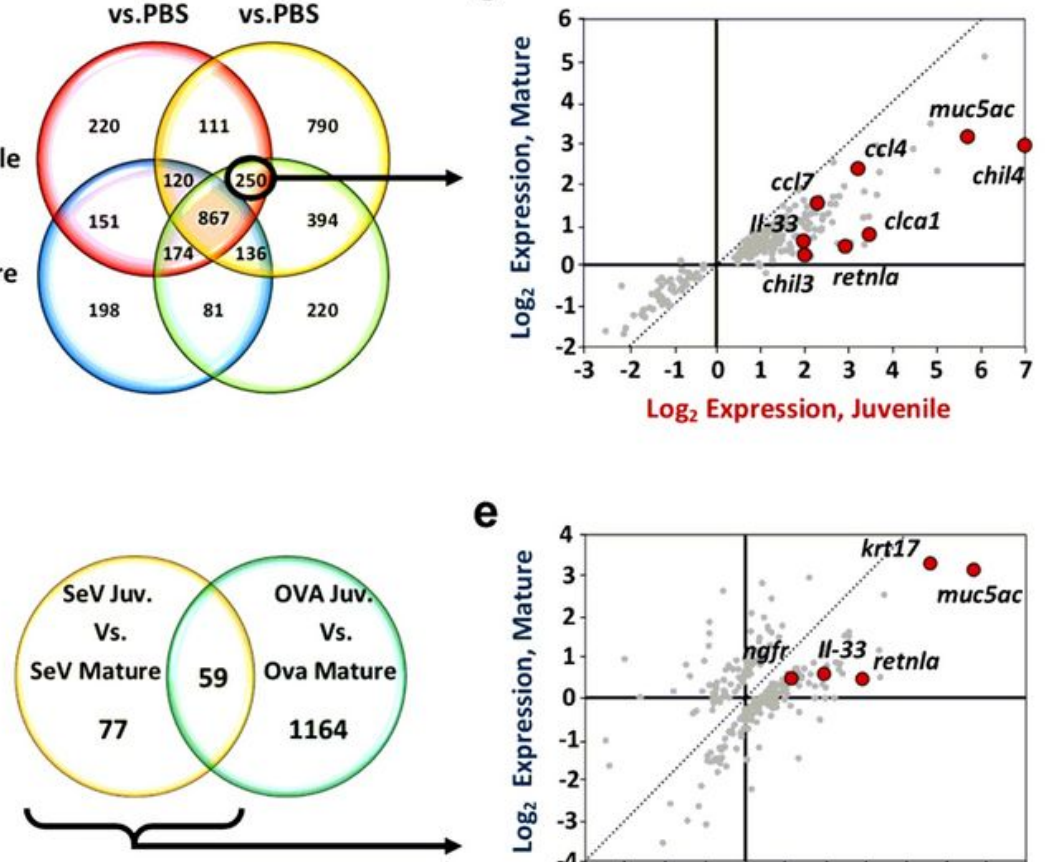

e

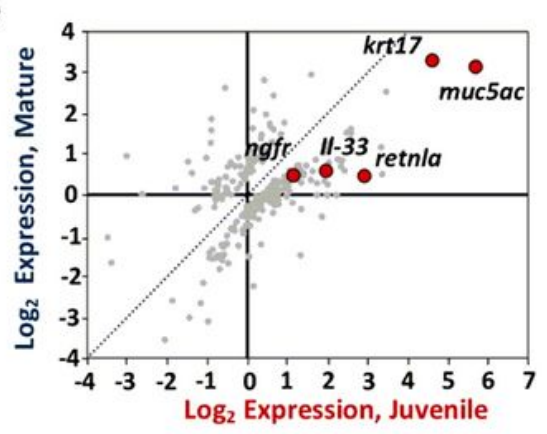

h

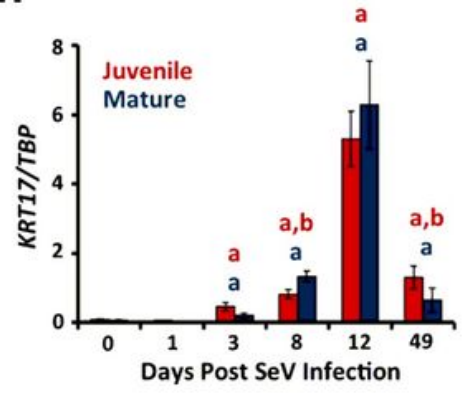

i

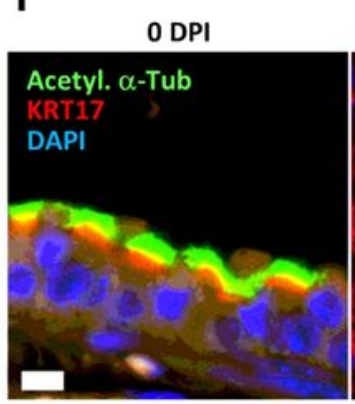

$12 \mathrm{DPI}$

$49 \mathrm{DPI}$
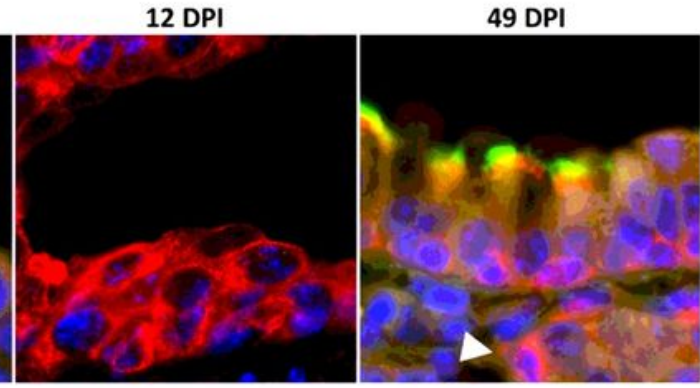

Figure 4

(see Figure Caption file in Supplementary Files) 
a

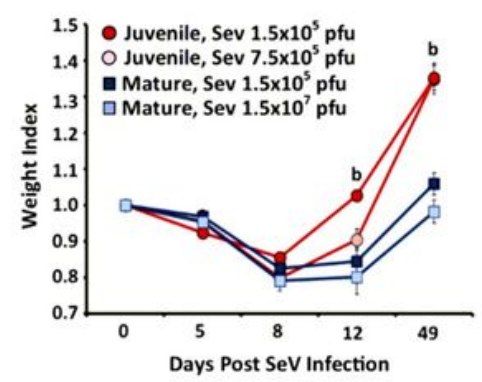

d

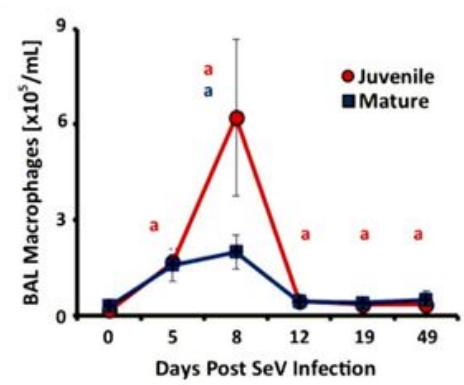

g

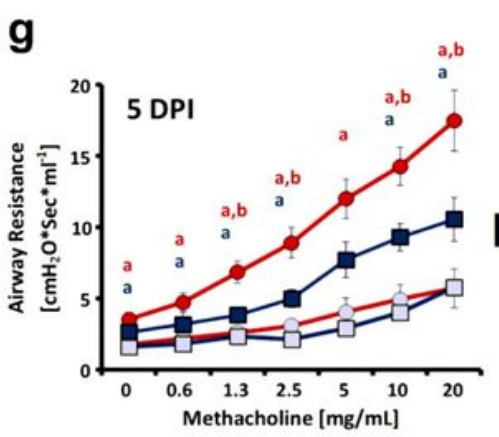

j

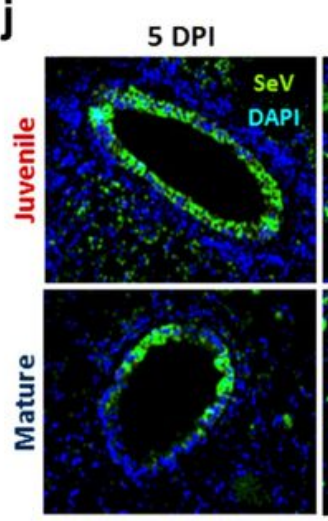

b

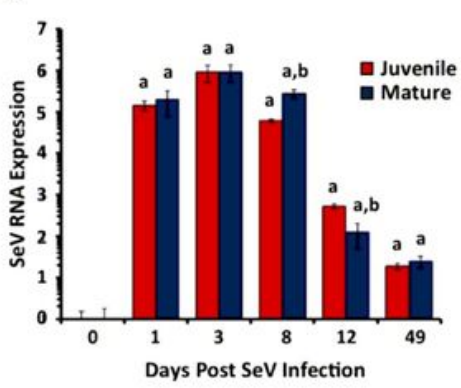

e

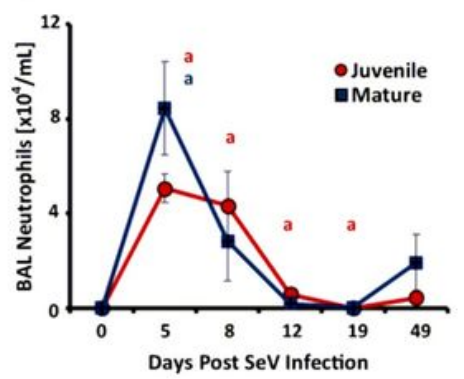

h

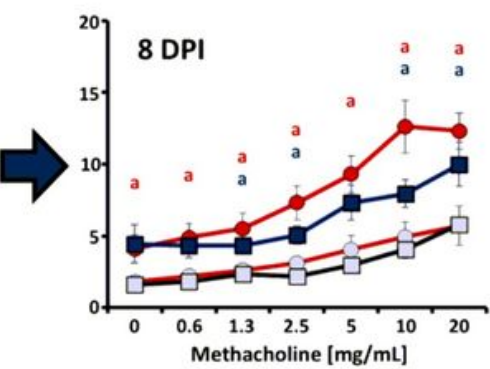

12 DPI

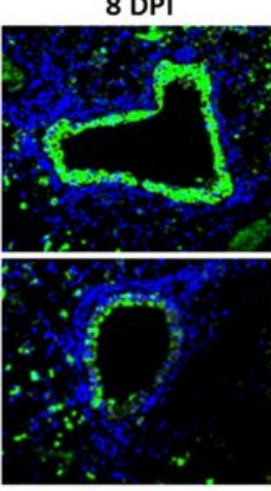

C

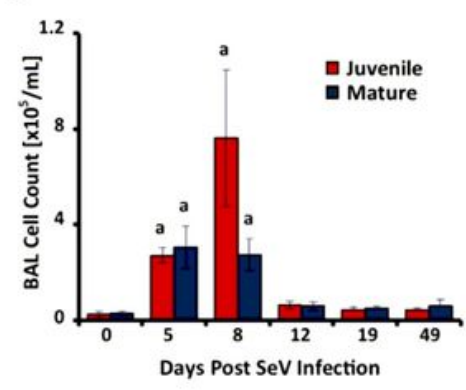

f

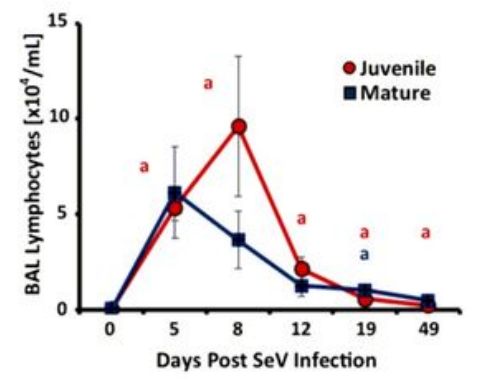

i

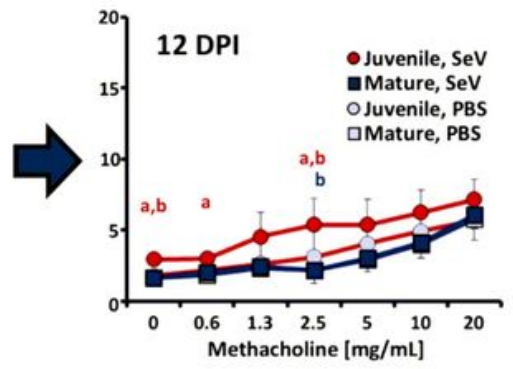

k

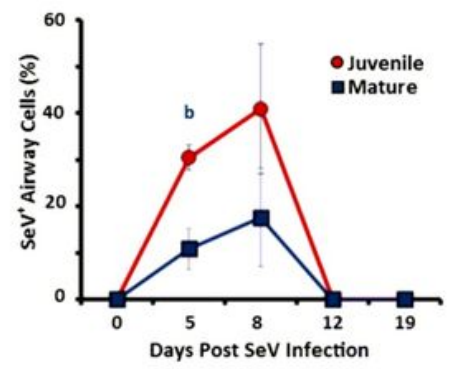

Figure 5

(see Figure Caption file in Supplementary Files) 
a

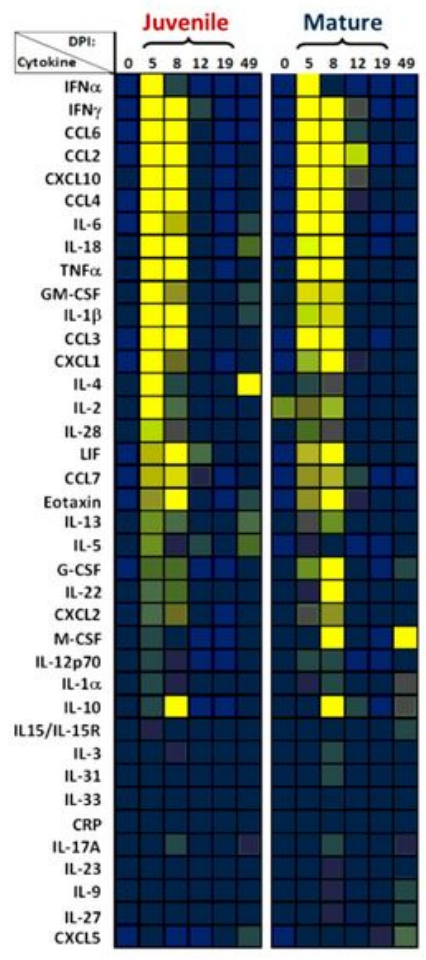

C

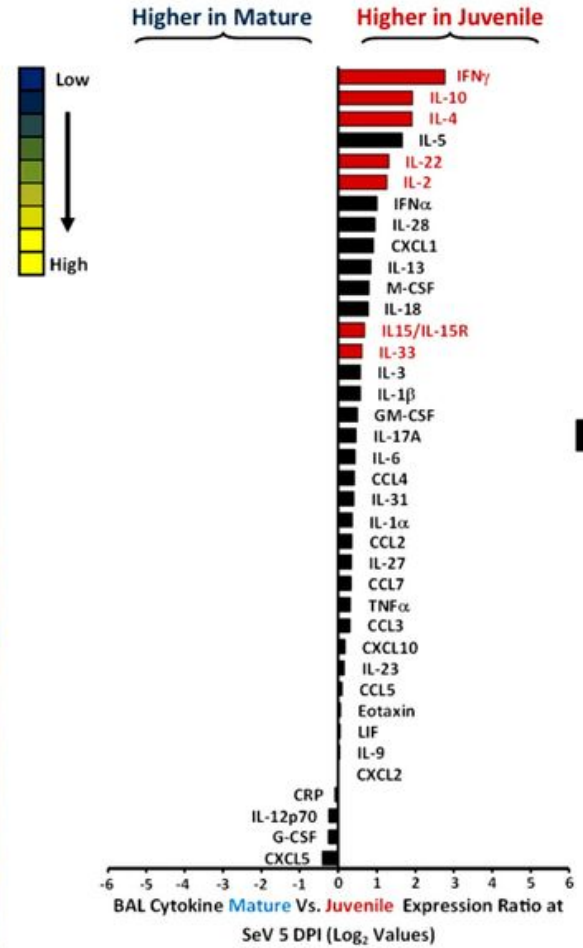

d

SeV 49 DPI

Higher in Mature Higher in Juvenile b

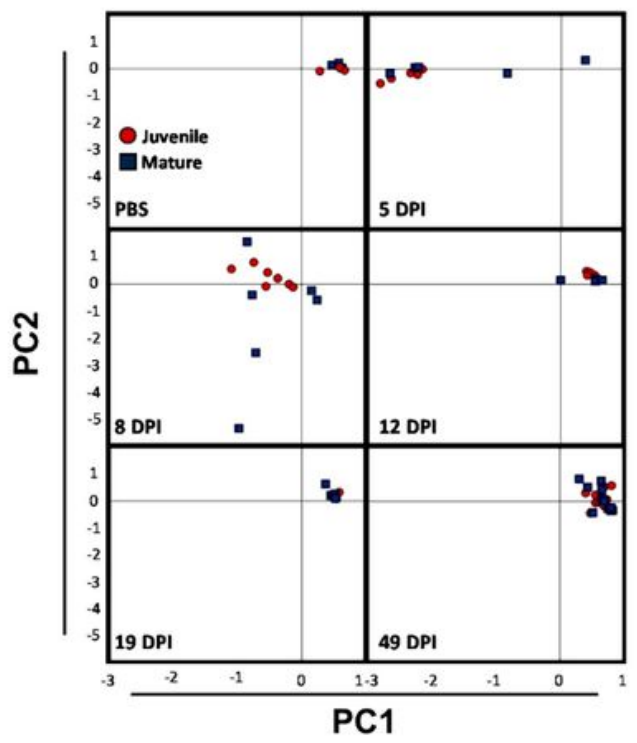

e

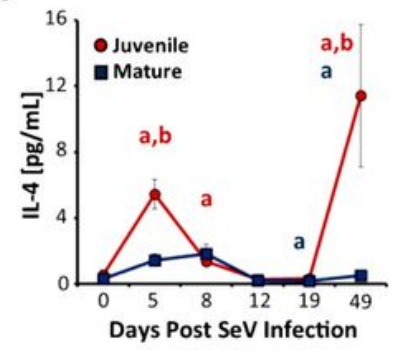

f

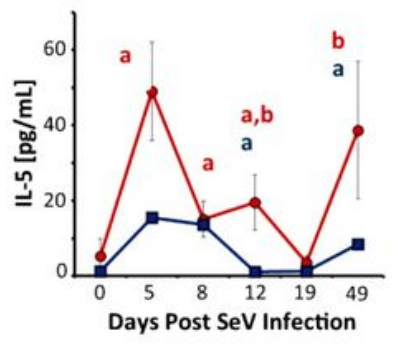

g

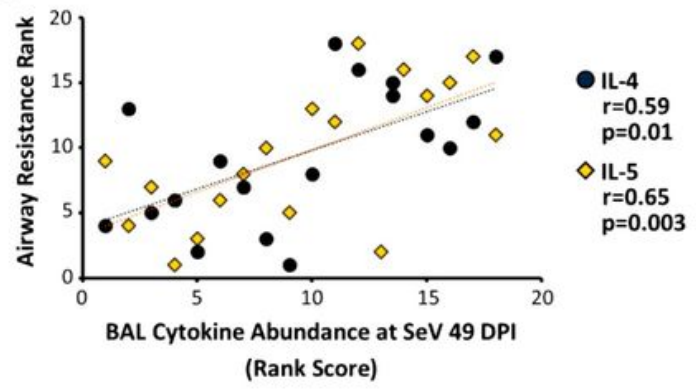

Figure 6

(see Figure Caption file in Supplementary Files) 
a
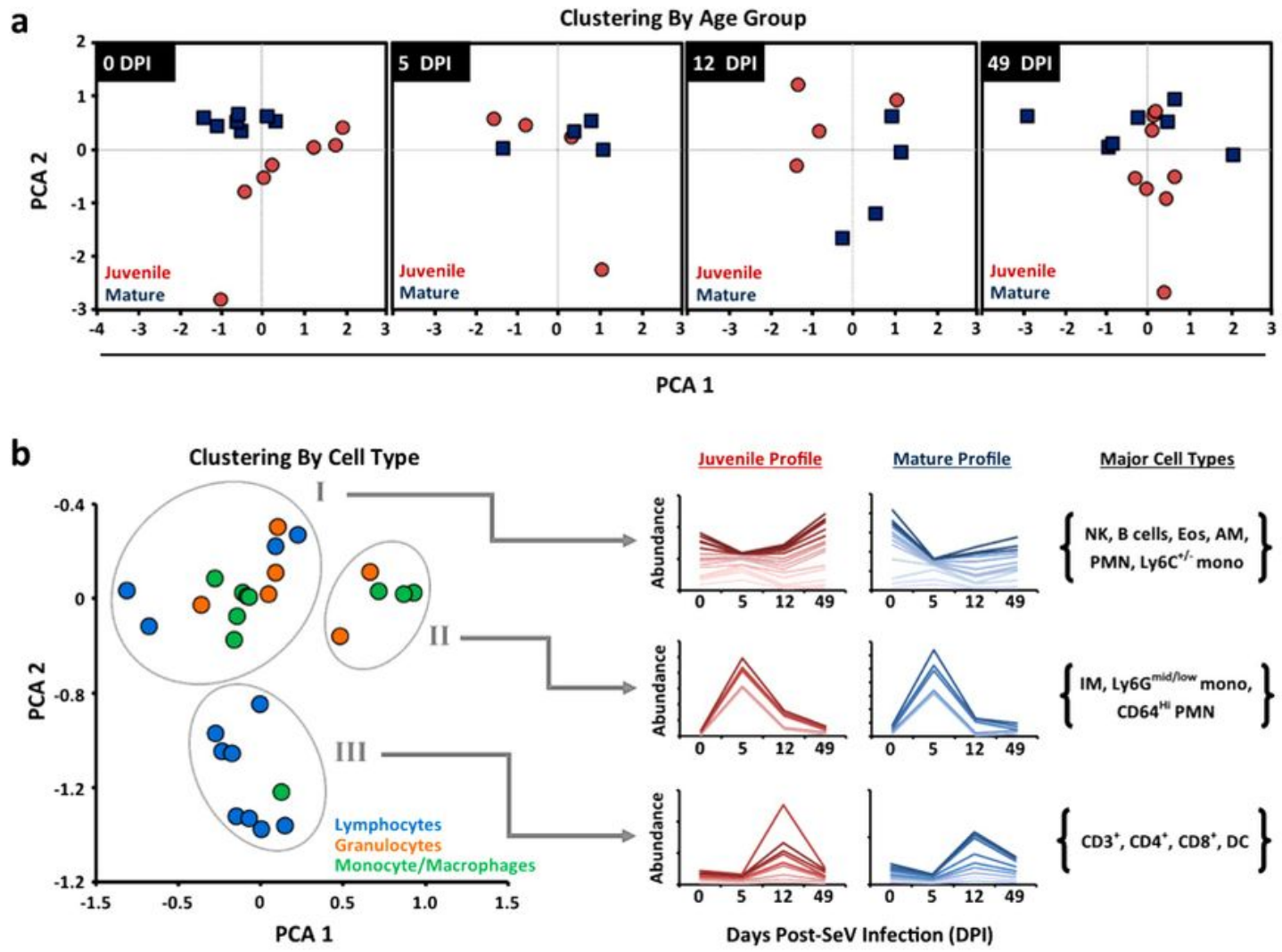

C

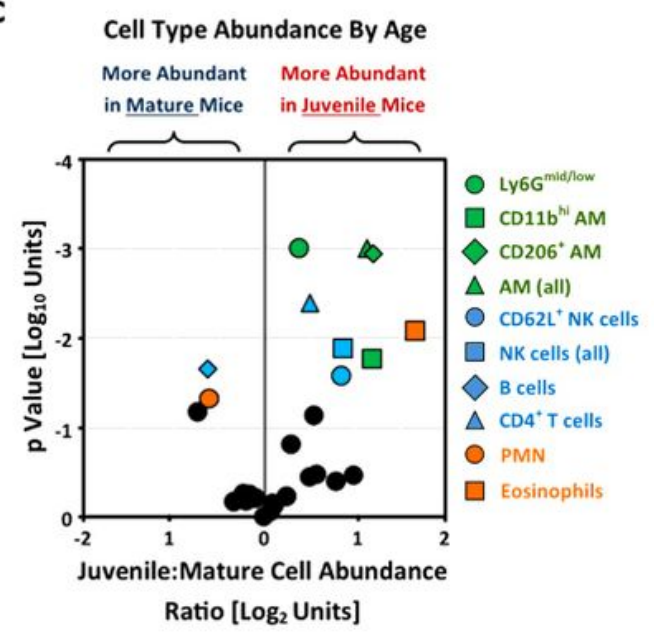

d

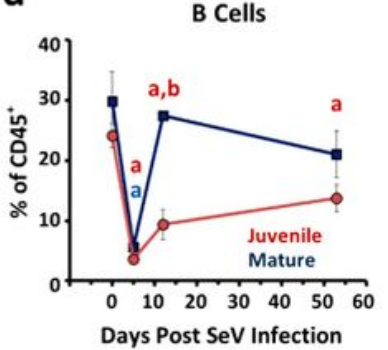

f

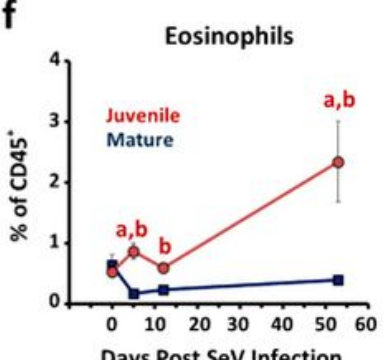

e PMNs

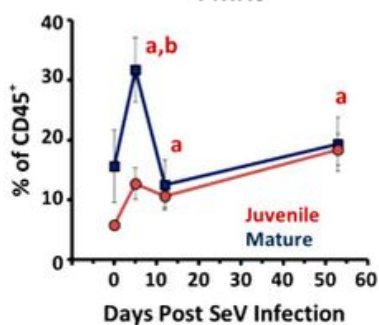

g

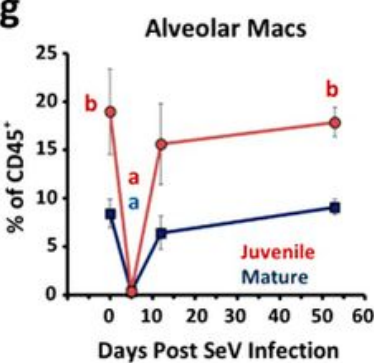

Figure 7

(see Figure Caption file in Supplementary Files) 
a
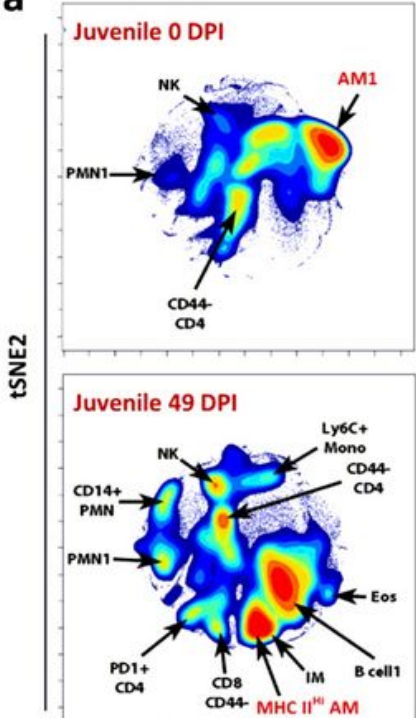

tSNE1

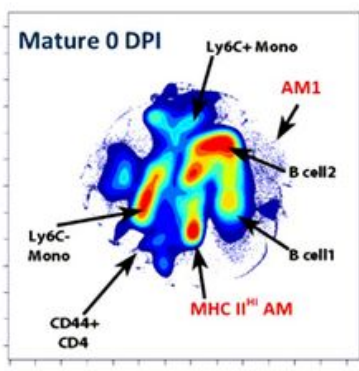

Mature $49 \mathrm{DPI}$

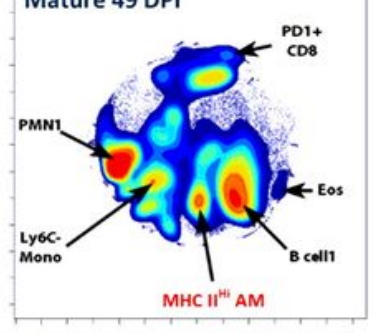

MHC ${ }^{\mathrm{HH}}$ AM b

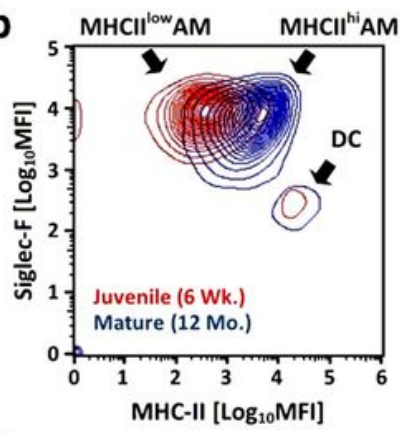

C

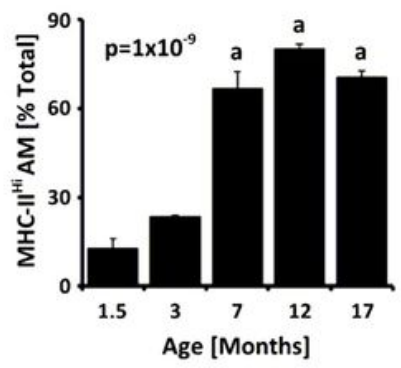

d

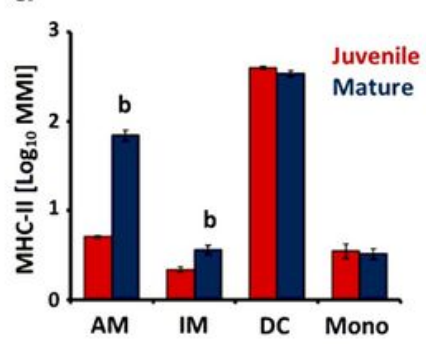

g

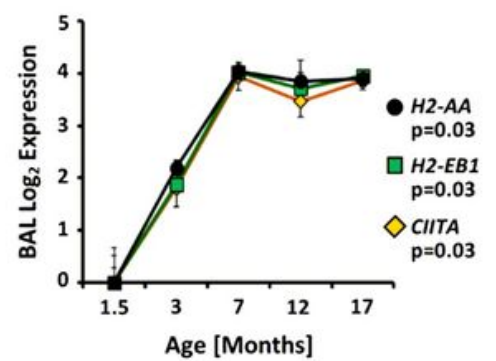

e

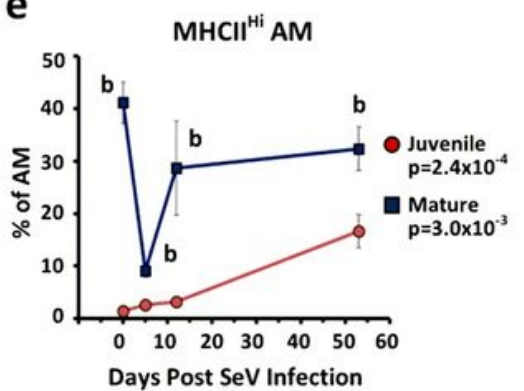

h Greater in Mature Greater in Juvenile

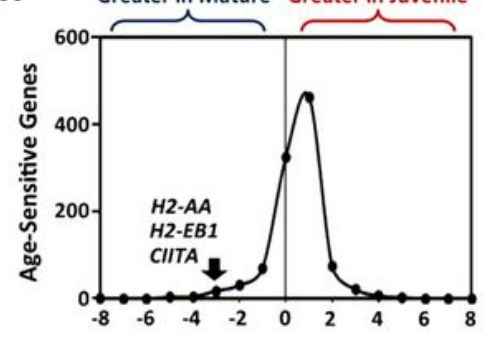

$\log _{2}$ Expression Ratio f

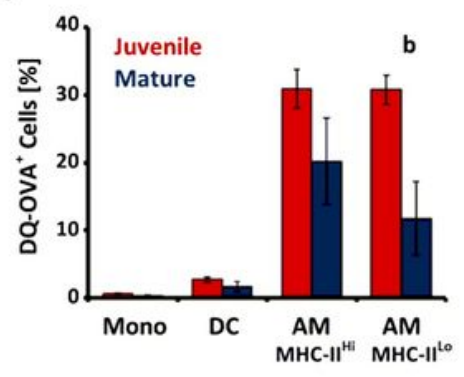

\begin{tabular}{|c|c|}
\hline KEGG Pathway & FDR \\
\hline mmu05310:Asthma & 0.004 \\
\hline mmu04145:Phagosome & 0.005 \\
\hline mmu05150:S. aureus infection & 0.005 \\
\hline $\begin{array}{l}\text { mmu04672:Immune network } \\
\text { for IgA production }\end{array}$ & 0.007 \\
\hline $\begin{array}{l}\text { mmu04612:Antigen processing } \\
\text { and presentation }\end{array}$ & 0.008 \\
\hline $\begin{array}{l}\text { mmu05321:Inflammatory } \\
\text { bowel disease (IBD) }\end{array}$ & 0.009 \\
\hline mmu05140:Leishmaniasis & 0.015 \\
\hline $\begin{array}{l}\text { mmu05332:Graft-versus-host } \\
\text { disease }\end{array}$ & 0.015 \\
\hline mmu05330:Allograft rejection & 0.022 \\
\hline
\end{tabular}

Figure 8

(see Figure Caption file in Supplementary Files) 


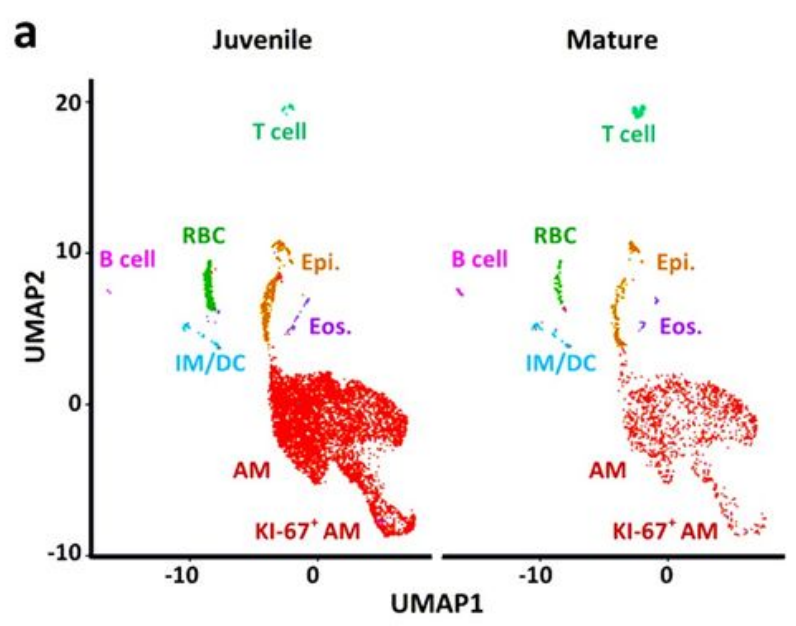

\begin{tabular}{|c|c|c|}
\hline Cell Type & $\begin{array}{l}\text { Juvenile } \\
\text { (n=7276) }\end{array}$ & $\begin{array}{l}\text { Mature } \\
(n=1616)\end{array}$ \\
\hline Alveolar Mac. (AM) & $69.83 \%$ & $62.91 \%$ \\
\hline $\mathrm{KI}-67^{+} \mathrm{AM}$ & $13.30 \%$ & $5.47 \%$ \\
\hline Epithelial & $7.26 \%$ & $11.39 \%$ \\
\hline Erythroid (RBC) & $3.90 \%$ & $3.03 \%$ \\
\hline T-Cell & $1.79 \%$ & $6.62 \%$ \\
\hline IM/DC & $1.54 \%$ & $3.90 \%$ \\
\hline Eosinophils (Eos) & $1.47 \%$ & $2.72 \%$ \\
\hline B-cell & $0.91 \%$ & $3.96 \%$ \\
\hline
\end{tabular}
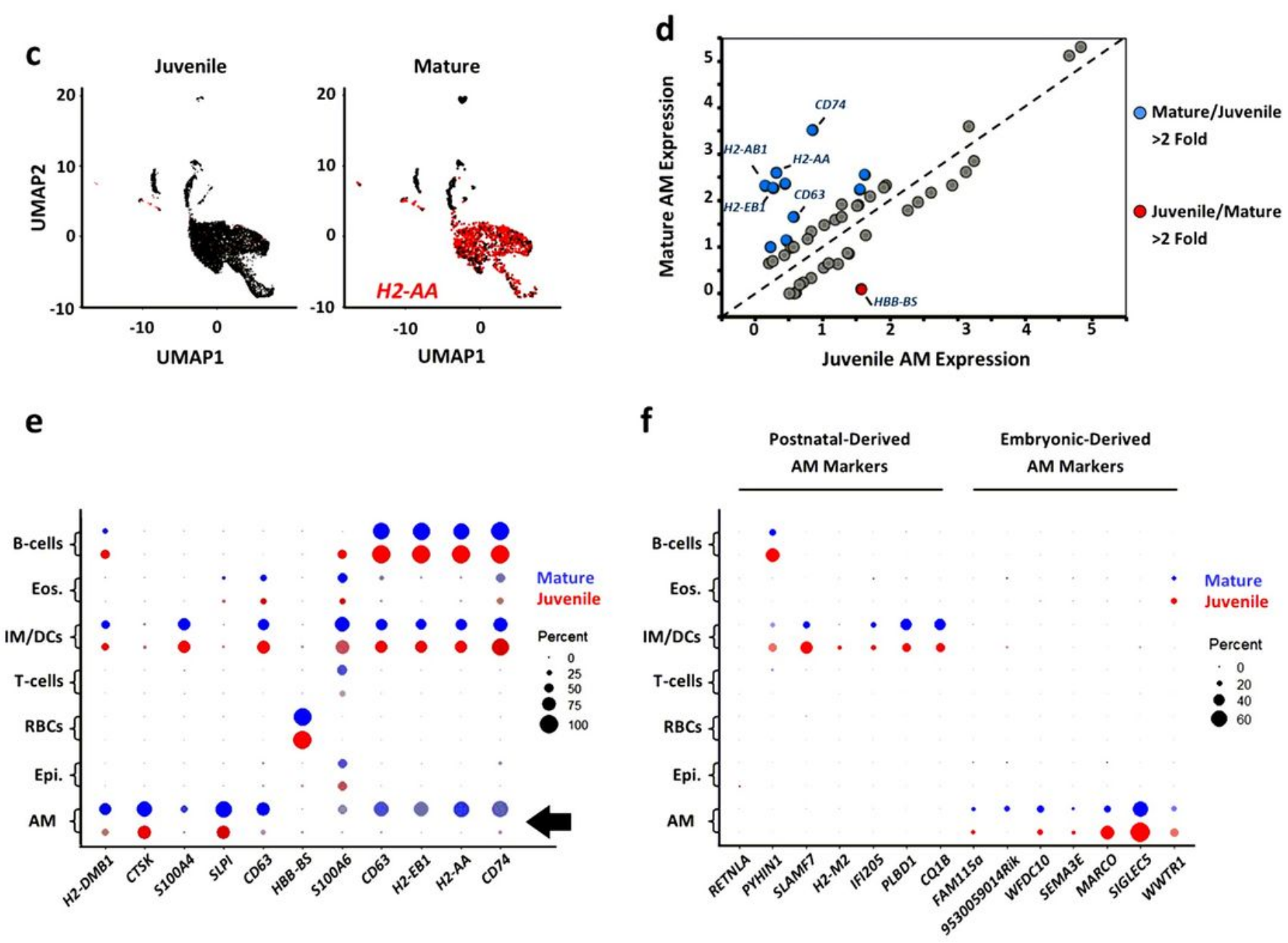

Figure 9

(see Figure Caption file in Supplementary Files) 
a
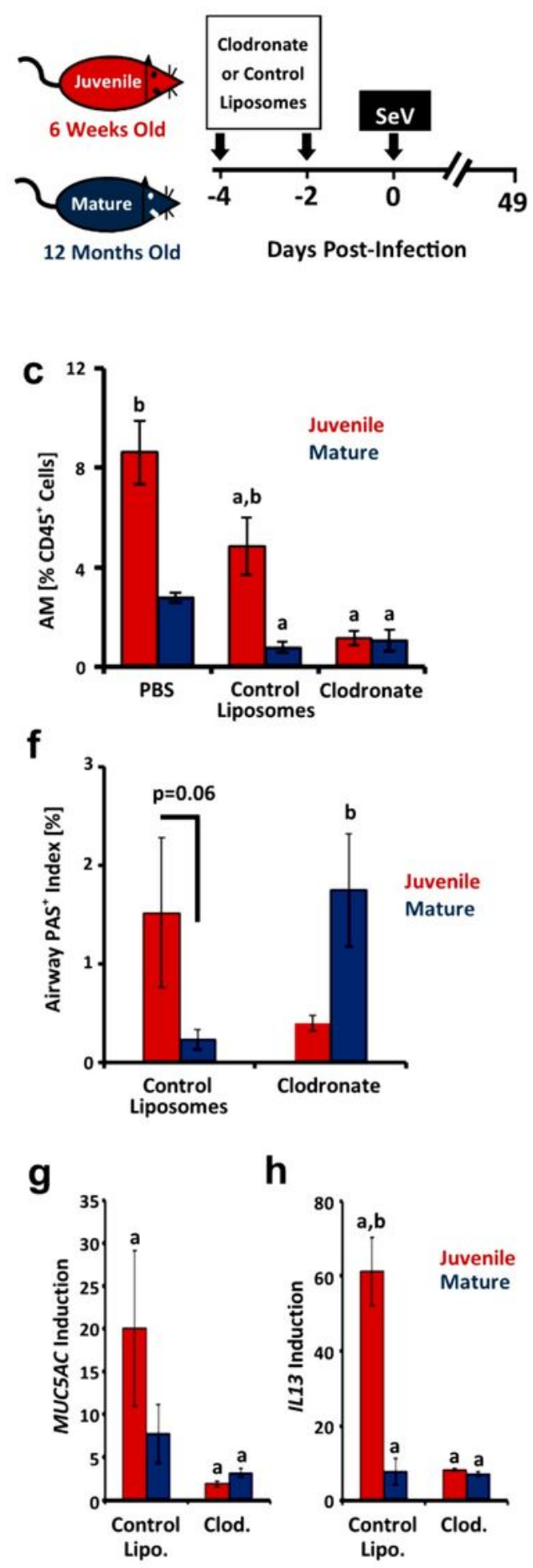

b

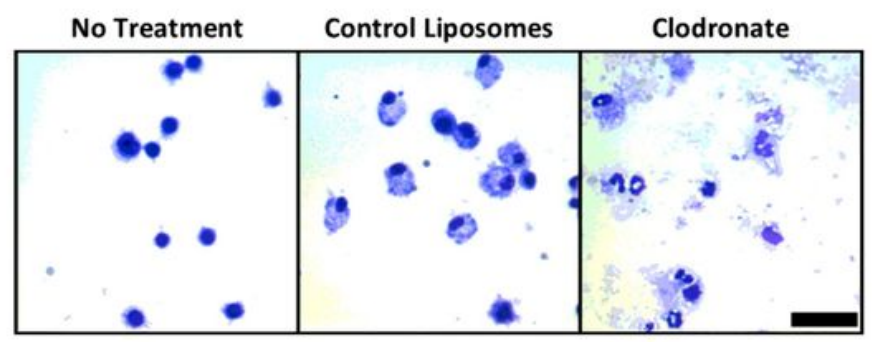

d
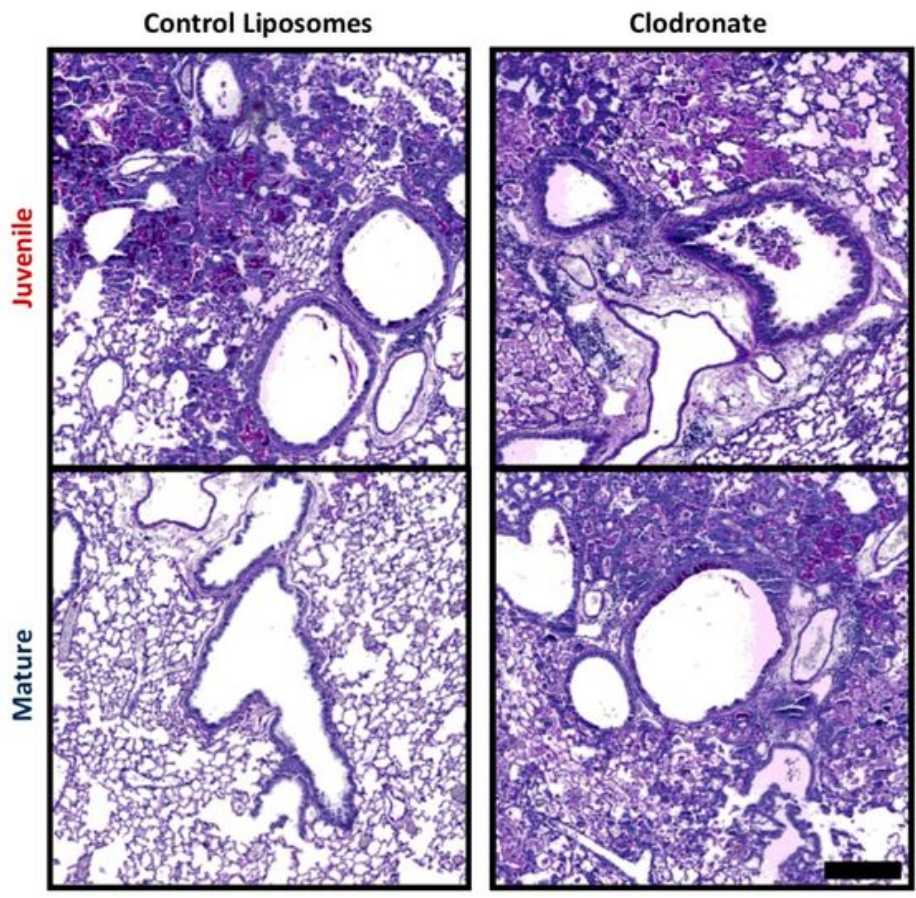

e
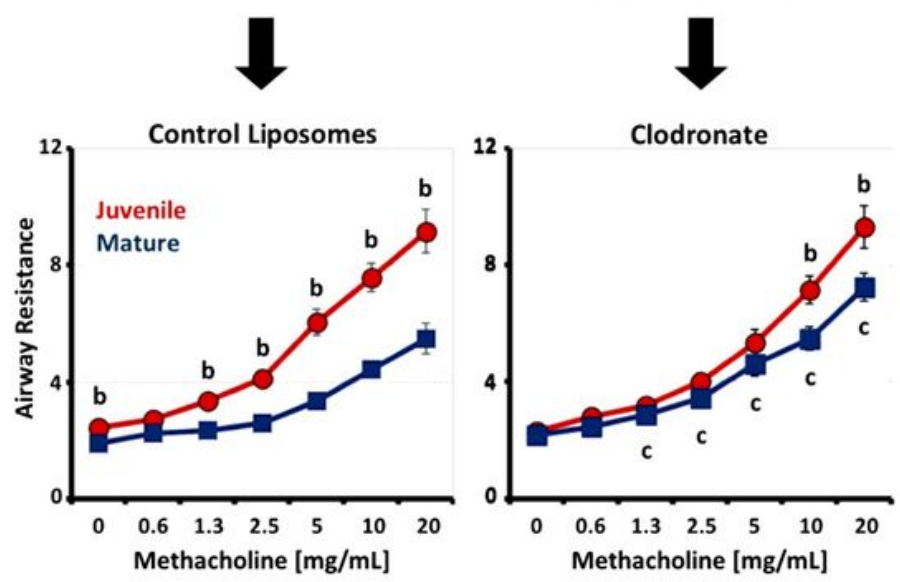

Figure 10

(see Figure Caption file in Supplementary Files)

\section{Supplementary Files}

This is a list of supplementary files associated with this preprint. Click to download. 
- Tables1NCOMM.docx

- Tables2NCOMM.docx

- Tables3NCOMM.docx

- Tables4NCOMM.docx

- FigureS1WL.tif

- FigureS2WLedit.tif

- Figures3WL.tif

- FigureS4WL.tif

- FigureS5WL.tif

- FigureS6WL.tif

- FigureS7WL.tif

- SupplementaryData1.xlsx

- SupplementaryData2.xIsx

- FigureCaptions.pdf 